\title{
Simulation of Shallow Cumuli and Their Transition to Deep Convective Clouds by Cloud-Resolving Models with Different Third-order Turbulence Closures
}

\author{
Anning Cheng ${ }^{1,2}$ and Kuan-Man $\mathrm{Xu}^{1}$
}

1. Atmospheric Sciences, NASA Langley Research Center, Hampton, VA

2. Center for Atmospheric Sciences, Hampton University, Hampton, VA

Submitted to

Quart. J. Roy. Meteor. Soc.

February 25, 2005

Corresponding author address:

Dr. Anning Cheng

Mail Stop 420

NASA Langley Research Center

Hampton, VA 23681

E-mail: a.cheng@larc.nasa.gov 


\section{ABSTRACT}

The abilities of cloud-resolving models (CRMs) with the double-Gaussian based and the single-Gaussian based third-order closures (TOCs) to simulate the shallow cumuli and their transition to deep convective clouds are compared in this study. The single-Gaussian based TOC is fully prognostic (FP), while the double-Gaussian based TOC is partially prognostic (PP). The latter only predicts three important third-order moments while the former predicts all the thirdorder moments. A shallow cumulus case is simulated by single-column versions of the FP and PP TOC models. The PP TOC improves the simulation of shallow cumulus greatly over the FP TOC by producing more realistic cloud structures. Large differences between the FP and PP TOC simulations appear in the cloud layer of the second- and third-order moments, which are related mainly to the underestimate of the cloud height in the FP TOC simulation. Sensitivity experiments and analysis of probability density functions (PDFs) used in the TOCs show that both the turbulence-scale condensation and higher-order moments are important to realistic simulations of the boundary-layer shallow cumuli.

A shallow to deep convective cloud transition case is also simulated by the 2-D versions of the FP and PP TOC models. Both CRMs can capture the transition from the shallow cumuli to deep convective clouds. The PP simulations produce more and deeper shallow cumuli than the FP simulations, but the FP simulations produce larger and wider convective clouds than the PP simulations. The temporal evolutions of cloud and precipitation are closely related to the turbulent transport, the cold pool and the cloud-scale circulation. The large amount of turbulent mixing associated with the shallow cumuli slows down the increase of the convective available potential energy and inhibits the early transition to deep convective clouds in the PP simulation. When the deep convective clouds fully develop and the precipitation is produced, the cold pools produced by the evaporation of the precipitation are not favorable to the formation of shallow cumuli.

KEYWORDS: Third-order turbulence closure, Shallow cumulus, Deep convective clouds 


\section{Introduction}

Cloud models with higher-order turbulence closures have been used to simulate convective planetary boundary layer (PBL) clouds. Despite the difficulties involved in the closure for higher-order moments, pressure terms and dissipation terms, higher-order closure models have been shown to be able to simulate a wide range of boundary layers. Using a third-order closure (TOC) model which predicts all third-order moments and diagnoses all fourth-order moments, André et al. (1976a,b) successfully simulated penetrative convection observed in a laboratory experiment. André at al. (1978) used the same model to simulate the 24-hour evolution of the mean and turbulent structures of the clear convective PBL observed during the Wangara experiment (Clark et al. 1971). The computed daytime and nocturnal structure quantitatively agreed with a number of experimental data.

Fully prognostic (FP) TOC models have been used to simulate PBL clouds with probability distribution function (PDF) based turbulence-scale condensation schemes (e.g. Sommeria and Deardorff 1977; Mellor 1977). FP TOC predicts all the third-order moments. Bougeault $(1981 \mathrm{a}, \mathrm{b})$ investigated the effects of different turbulence-scale condensation parameterizations on the simulation of tradewind cumulus. A simple, skewed exponential distribution was found to be most efficient for parameterizing the TKE buoyancy production. However, the simple skewed exponential scheme does not have a variable skewness to represent diverse PBL clouds ranging from shallow cumulus, stratocumulus to solid stratus. Krueger (1988) developed a two-dimensional (2-D) cloud resolving model (CRM) that couples cloud-scale dynamics with a TOC that parameterizes turbulent processes in the boundary layer and in clouds. This TOC used a quasi-Gaussian closure (single-Gaussian based closure) to express fourth-order moments in terms of second-order moments and the clipping approximation to dampen the unrealistic growth of the triple correlations because of the use of the quasi-Gaussian closure. The

turbulence-scale condensation scheme was based upon the single-Gaussian distribution. This model was used to simulate BOMEX (Barbados Oceanographic and Meteorological EXperiment) 
marine shallow cumuli (Krueger and Bergeron 1994) and was able to simulate individual shallow cumuli. A shortcoming of their model was seen in the simulated profile of the mean cloud fraction. The cloud fraction was underestimated near the cloud base and had its largest value near the cloud top.

To obtain a realistic simulation of various types of PBL clouds, the internal physical consistency and the flexibility of TOC models must be addressed. In Bougeault's (1981a,b) TOC model, the fourth-order moments were expressed by using the quasi-Gaussian closure, but buoyancy production terms involving liquid water were parameterized empirically based upon large-eddy simulation (LES) data. Recently, Lappen and Randall (2001a,b) developed a minimally-prognostic (MP) TOC, which predicts the triple correlation of vertical velocity $\left(\overline{w^{\prime 3}}\right)$ only. They combined the mass flux closure (e.g. Arakawa 1969; Betts 1973; Albrecht 1979; Randall 1987) with a higher-order closure. A physically-based method for diagnosing the updraft area and the convective mass flux was developed using the second- and third-order moments of the vertical velocity. Golaz et al. (2002a,b; hereafter GLC) developed an MP TOC using a doubleGaussian based closure. There are various kinds of double Gaussians (e.g. Lewellen and Yoh 1993; hereafter, the LY double Gaussian; Larson et al. 2002). They used the analytic double Gaussian I proposed by Larson et al. (2002). GLC also used a few assumptions to determine the magnitude of each Gaussian. All the moments in this approach are based upon the same PDF so that the internal physical consistency is ensured. This model was not only capable of reproducing reasonable profiles from first-order moments to third-order moments, but also simulating a reasonable time evolution of cloud fraction. Two shortcomings of the GLC model are that the third-order moment of liquid-water potential temperature $\left(\overline{\theta_{l}^{\prime 3}}\right)$ was assumed to be zero and the third-order moment of total water mixing ratio $\left(\overline{q_{t}^{\prime 3}}\right)$ was assumed to be proportional to $\overline{w^{\prime 3}}$.

Cheng et al. (2004) recently compared the ability of the FP and MP TOC models to simulate shallow cumuli. They found that an unrealistically strong liquid water oscillation (LWO) 
can exist in either model if the liquid-water buoyancy production terms in the third-order moment equations are not parameterized properly. The FP model produced a stronger LWO than the MP model because it has more equations with buoyancy production terms. Since the predictive equations of $\overline{\theta_{l}^{\prime 3}}$ and $\overline{q_{t}^{\prime 3}}$ do not contain the buoyancy production term, Cheng et al. (2004) surmised that a partially-prognostic (PP) TOC model, which predicts $\overline{w^{\prime 3}}, \overline{\theta_{l}^{\prime 3}}$ and $\overline{q_{t}^{\prime 3}}$, but diagnoses the rest of the third-order moments, will not produce an LWO as large as that seen in the FP model.

One of the purposes of this study is to evaluate the performance of a newly developed PP TOC model. In the PP model, $\overline{w^{\prime 3}}, \overline{\theta_{l}^{\prime 3}}$, and $\overline{q_{t}^{\prime^{3}}}$ are predicted and the rest of the third-order moments and all fourth-order moments are diagnostically obtained using a double-Gaussian PDF. Another purpose of this paper is to compare the abilities of CRMs with the FP and PP TOCs to simulate shallow cumuli and their transition to deep convective clouds. The questions that this paper will try to answer are 1) How do the turbulence-scale condensation and higher-order moments affect the shallow cumulus simulations? 2) How do the different TOCs affect the transition from shallow cumuli to deep convective clouds? and 3) How do the shallow cumuli and deep convective clouds interact?

The rest of the paper is organized as follows. Section 2 gives a description of the PP TOC model. Section 3 compares the ability of the FP and PP TOCs to simulate a shallow cumulus case and shows the importance of the higher-order moments and the turbulence-scale condensation scheme with two sensitivity experiments. Section 4 explores the effects of different TOCs on the simulation of the transition from shallow cumuli to deep convective clouds and the interactions between shallow cumuli and deep convective clouds. Summary and discussions are provided in Section 5 . 


\section{Model description}

The University of California-Los Angeles (UCLA)/Langley Research Center (LaRC) CRM is used in this study. This model was previously known as the UCLA/Colorado State University CRM (Krueger 1988; Xu and Randall 1995). A PP TOC was implemented in the model in addition to the original FP TOC.

The PDF is an important component of the PP version of the CRM because the higherorder moments are all diagnosed from the same PDF to ensure internal consistency, the cloud fraction and liquid water, and the buoyancy production terms of the second- and third-order moment equations are also derived from the PDF. Double Gaussian PDFs are able to describe the PBL clouds from observations (e.g., LY; Larson et al. 2002) and models (e.g., GLC). In this study, we used the analytical double-Gaussian II PDF proposed by Larson et al. (2002) because this PDF reduces to a single-Gaussian when the skewnesses of $\theta_{l}$ and $q_{t}$ go to zero. Also, unlike the analytical double-Gaussian I PDF (Larson et al. 2002), the variances of the vertical velocity of the two Gaussians are not required to be equal.

In the PP model, the same first- and second-order moment equations and $\overline{w^{\prime 3}}$ equation as in GLC are used except that the third-order moment equations of $\theta_{l}$ and $q_{t}$ are added in order to, as mentioned earlier, remove the key assumptions on $\overline{\theta^{\prime}{ }_{l}^{3}}$ and $\overline{q^{\prime}{ }_{t}^{3}}$ used in GLC. That is,

$$
\frac{\partial \overline{\alpha^{\prime}}}{\partial t}=3 \overline{\alpha^{\prime 2}} \frac{\partial \overline{w^{\prime} \alpha^{\prime}}}{\partial z}-3 \overline{w^{\prime} \alpha^{\prime 2}} \frac{\partial \bar{\alpha}}{\partial z}-\varepsilon_{\alpha \alpha \alpha}+K \nabla^{2} \overline{\alpha^{\prime 3}}-\frac{\partial \overline{w^{\prime} \alpha^{\prime 3}}}{\partial z}
$$

where $\alpha$ can be $\theta_{l}$ or $q_{t} \cdot \varepsilon_{\alpha \alpha \alpha}=\frac{c_{10}}{\tau} \overline{\alpha^{\prime 3}}$, where $c_{10}=3.12$ is a constant, and $\tau$ is the dissipation time scale (please see GLC for details about this time scale). $K=15 \mathrm{~m}^{2} \mathrm{~s}^{-1}$ is the diffusion coefficient and $\nabla^{2}=\partial^{2} / \partial x^{2}+\partial^{2} / \partial y^{2}+\partial^{2} / \partial z^{2}$. The terms on the right hand side are 
the transport, the mean gradient, the dissipation, the diffusion and the fourth-order terms, respectively. The fourth-order term can be calculated by

$$
\begin{gathered}
\overline{w^{\prime} \alpha^{\prime}}=a \sigma_{w 1}^{2} \sigma_{\alpha 1}^{2}\left(3 r_{w \alpha 1}+3 \tilde{w}_{1} \tilde{\alpha}_{1}+3 r_{w \alpha 1} \tilde{\alpha}_{1}^{2}+\tilde{w}_{1} \tilde{\alpha}_{1}^{3}\right)+ \\
(1-a) \sigma_{w 2}^{2} \sigma_{\alpha 2}^{2}\left(3 r_{w \alpha 2}+3 \tilde{w}_{2} \tilde{\alpha}_{2}+3 r_{w \alpha 2} \tilde{\alpha}_{2}^{2}+\tilde{w}_{2} \tilde{\alpha}_{2}^{3}\right)
\end{gathered}
$$

where subscript 1 and 2 represent the first plume (Gaussian) and the second plume, respectively. $a$ is the magnitude of the first plume, $\sigma$ is the standard deviation, and $r$ is the within-plume correlation. Any normalized variable is calculated by $\tilde{\chi}_{i}=\left(\chi_{i}-\bar{\chi}\right) / \sigma_{\chi i}$, where $\chi$ can be $w, \theta_{l}$ or $q_{t}$, and $i$ can be 1 or 2 . Formula (2) can be derived by integrating the fourth-order moments over the double-Gaussian PDF. We can see that the fourth-order moments are closely related to the standard deviation of each plume.

All equations are discretized on a vertically staggered C-grid. In such a grid, terms such as $\bar{w} \partial \overline{w^{\prime 2}} / \partial z$ can be discretized without any interpolation. Turbulence advection terms, such as

$\partial \overline{w^{\prime 3}} / \partial z$ and $\partial \overline{w^{\prime 4}} / \partial z$ appearing in the predictive equations for $\overline{w^{\prime 2}}$ and $\overline{w^{\prime 3}}$, respectively, can also be calculated directly. The production terms such as $\overline{w^{\prime 2}} \partial \overline{q_{t}} / \partial z$ have to be interpolated. The parameters associated with the PDF are located at the mid-layer points. A detailed description of various elements of the model can be found in GLC, except for the aforementioned differences.

\section{Shallow cumulus case}

\subsection{Design of experiment}

BOMEX took place in June 1969. The observed cloud fraction was about $10 \%$. It is a purely shallow cumulus case. The horizontal scale of most individual cumulus clouds is less than $1 \mathrm{~km}$. The cloud layer has a much lower relative humidity than that observed in stratocumulus 
cloud layers. It is difficult for 1-D and 2-D CRMs to capture the vertical structure of shallow cumuli if turbulence is not properly parameterized.

The BOMEX case is run with a vertical resolution of $40 \mathrm{~m}$ in a domain that extends from 0 to $3000 \mathrm{~m}$ by the single column version of the UCLA/LaRC CRM. We use a time step of $2 \mathrm{~s}$. The length of the simulation is $6 \mathrm{~h}$. The surface sensible heat and latent heat fluxes are prescribed at $9.5 \mathrm{~W} \mathrm{~m}^{-2}$ and $153.4 \mathrm{~W} \mathrm{~m}^{-2}$, respectively. The radiative cooling, large scale subsidence, and horizontal moisture advection are also prescribed. Details of the model configuration are the same as those described in Siebesma et al. (2003) for an LES intercomparison study. The LESintercomparison data are used for comparison in this study. When the LES-intercomparison data are not available, those from the UCLA-LES (Stevens et al. 1999) are used.

\subsection{Results}

The computational cost of the PP model is about a half of that of the FP model, taking $66 \mathrm{~s}$ and $128 \mathrm{~s}$ on a $1.3 \mathrm{GHz}$ notebook to run the $6 \mathrm{~h}$ simulations, respectively. This means that about one half of the computational time is used for the third-order moment equations in the FP model.

A one-dimensional boundary-layer model needs to reasonably represent the mean profiles of potential temperature and water vapor mixing ratio, the distributions of cloud fraction and liquid water for cloudy boundary-layers, the vertical heat and moisture fluxes, the variances of vertical velocity, potential temperature and total water mixing ratio, the various terms in the TKE equation, and the skewnesses of vertical velocity, potential temperature, and total water mixing ratio. All these aspects are related to each other. For example, if a model underestimates the skewnesses of potential temperature and water vapor mixing ratio, the cloud fraction will be overestimated. If the TKE is underestimated, vertical transports of heat and moisture will be underestimated.

The vertical distribution of clouds is an important indicator of the success of boundary layer models. The cloud fraction for tradewind cumulus is relatively large near the cloud base but 
small near the cloud top (Fig. Ia). It can be seen that the magnitude and distribution of cloud fraction and cloud top and base heights are quite different between the two TOC models (Figs. $\mathbf{l b}, \mathrm{c})$. The cloud fraction from the PP model is much closer to that of the UCLA-LES and within the range of LES-simulated cloud fractions (Siebesma et al. 2003). The maximum cloud fraction from the FP model is about $50 \%$, which is much larger than that simulated by the UCLA-LES (Fig. $\mathbf{l}$ a). Another result appearing in Fig. $\boldsymbol{l}$ is that the PP model simulates a quantitatively correct structure of tradewind cumuli, with the largest cloud fraction of about $6 \%$ near the cloud base, as in LESs. The FP model has its largest cloud fraction near the cloud top, which is similar to the result of Krueger and Bergeron (1994). This difference between the PP and FP models is closely related to the skewnesses of $\theta_{l}$ and $q_{t}$, as will be discussed later.

The differences between the PP model and the LESs are much smaller than those between the FP model and the LESs in all second-order moments shown in Figs. 2 and 3. The secondorder moment of vertical velocity $\left(\overline{w^{\prime} w^{\prime}}\right)$ averaged between hours 3 and 5 of the BOMEX simulations are compared with the mean and standard derivations of the eleven LESs (Siebesma et al. 2003). There are two peaks in the LES consensus, one in the subcloud layer and the other within the upper part of the cloud layer, and there is a $\overline{w^{\prime} w^{\prime}}$ minimum near the cloud base between the two peaks. There are no significant differences of $\overline{w^{\prime} w^{\prime}}$ in the subcloud layer between the TOC models, but there are significant differences in the cloud layer. Although the PP model agrees well with the LES in the cloud layer, $\overline{w^{\prime} w^{\prime}}$ is underestimated significantly near the cloud top. The vertical profiles of $\overline{\theta_{l}^{\prime 2}}, \overline{q_{t}^{\prime 2}}$ and $\overline{q_{t}^{\prime} \theta_{l}^{\prime}}$ are compared with those from the UCLA-LES (Figs. 2b, c, and d). Their peak magnitudes are approximately the same between the two TOC models. The PP model captures the vertical structure of these second-order moments better than the FP model because the peaks in the cloud layer are much lower in the FP model compared to the LES. The underestimate of the cloud layer depth in the FP model is the primary reason. The 
vertical fluxes of liquid-water potential temperature and total water mixing ratio are shown in Fig. 3. It can also be seen that the differences between the PP model and the LES are much smaller than those between the FP model and LES.

The third-order moments $\left(\overline{w^{\prime 3}}, \overline{\theta_{l}^{\prime 3}}\right.$ and $\left.\overline{q_{t}^{\prime 3}}\right)$ are better predicted in the PP model than in the FP model (Fig. 4). There are two $\overline{w^{\prime 3}}$ peaks from the LES and the two TOC models, one in the subcloud layer and the other in the cloud layer. The value of $\overline{w^{\prime 3}}$ from the FP simulation is about one order of magnitude smaller than the PP simulation. $\overline{\theta_{l}^{\prime 3}}$ and $\overline{q_{t}^{\prime 3}}$ from the FP model, as expected, are almost zero, due to the single-Gaussian based closure. The third moments produced by the PP model have similar magnitudes and vertical distributions to the UCLA-LES although some differences are apparent. This suggest that there is room for further refinement of the PP model.

In summary, the PP model improves the simulations of shallow cumuli greatly over the FP model, in particular, reducing the magnitude of cloud fraction, correcting the unrealistic vertical distribution of cloud fraction and increasing the vertical extent of clouds. There are also large differences between the FP model and PP model for the second- and third-order moments. The third-order moments of $\theta_{l}, q_{t}$ and $w$ from the FP model are almost zero, while the second-order moments from the FP model have their peak value at lower levels than the LESs. These differences can be attributed to either the fourth-order moments, the turbulence-scale condensation or the buoyancy production terms for the higher-order moment equations since these are the only differences between the FP and PP models. In the following section, we will examine the effects of the fourth-order moments and the turbulence-scale condensation on the shallow cumulus simulation. The effects of the buoyancy production on the higher-order moment equations can be found in Cheng et al. (2004). 


\subsection{Effects of the fourth-order moments and the turbulence-scale condensation}

The FP model differs from the PP model in that it uses a single-Gaussian PDF for the turbulence-scale condensation, it predicts all third-order moments and diagnoses all fourth-order moments using the quasi-Gaussian assumption. In order to understand the importance of the PDF and higher-order moments in the TOC models, two experiments have been performed. In Experiment 1 (Exp. 1), we run the PP model except that its turbulence-scale condensation is replaced by the single-Gaussian formulation. In Experiment 2 (Exp. 2), we run the PP model while using the fourth-order moments based on the quasi-Gaussian approximation. That is, formula (2) is not used in this experiment, but the lower-order moments are obtained by the PP model, which are expected to be impacted by the inconsistent fourth-order moment closure.

The time-height cross sections of cloud fractions from Exps. 1 and 2 are shown in Fig. 5. Exp. 1 produces a cloud base height and a location of the maximum cloud fraction near the cloud base that are consistent with the UCLA-LES (Fig. 1 a), but the cloud fraction is too small and the cloud top is too low. Compared with the results from the PP model (Fig. $\mathbf{l}$ b), it can be concluded that the low cloud top may be caused by the single-Gaussian condensation scheme, suggesting that the skewness of PDF is important near the cloud top. On the other hand, Exp. 2 produces a cloud amount that is too high and its vertical distribution of cloud fraction is not similar to that of tradewind cumulus (Fig. 5b). These results are rather similar to those in the FP simulation $($ Fig. 1 c). Because the common attribute between the FP simulation and Exp. 2 is the quasi-Gaussian based fourth-order moment closure, the poor performance of the two simulations seems to be related to the higher-moment closure.

Fig. 6a shows the joint distributions of $\theta_{l}$ and $q_{t}$ diagnosed from the UCLA-LES at 780 $\mathrm{m}$ between hours 4 and 5. Fig. $6 \mathrm{~b}$ and $\mathrm{c}$ are the same distributions calculated from the doubleGaussian and single-Gaussian approaches using input from the UCLA-LES, respectively. The 
first- and second-order moments are needed to determine the joint distribution of $\theta_{l}$ and $q_{t}$ in the single-Gaussian approach, while the third-order moments are also needed to determine the distribution in the double-Gaussian approach. This particular level is chosen because there are large differences in cloud fractions there among the UCLA-LES, PP model and FP model. We can see the effects of the PDF formulation on the turbulence-scale condensation by comparing Fig. $\mathbf{6} \mathrm{b}$ and c. Both the single-Gaussian and the double Gaussian approaches diagnose about $10 \%$ cloud fractions since the PDFs are located mainly in the clear region of the diagrams $(\boldsymbol{F i g}$. 6 b, c). However, differences can still be seen from the diagrams. The joint distribution of $\theta_{l}$ and $q_{t}$ from the single-Gaussian approach is symmetric while that diagnosed from the UCLA-LES and that from the double-Gaussian approach are skewed. There are long tails in the cloudy region with joint normalized probability densities about 0.5 for both the UCLA-LES and the double-Gaussian approach, but greater than 1 for the single-Gaussian approach.

Fig. 7a and b are the same as Fig. 6 b and c, respectively, except the input is from the FP model. We can see that the joint distributions of $\theta_{l}$ and $q_{t}$ produced by the single-Gaussian and double-Gaussian approaches are generally similar. About a half of each PDF falls into the cloudy region of the diagrams, which means that both the single-Gaussian and double-Gaussian approaches predict cloud fractions of about $50 \%$.

Furthermore, it can be seen that the differences in cloud fraction are more pronoucedly caused by the higher-order moments than by the turbulence-scale condensation. This is because the differences between Fig. 6 and Fig. 7 are related to those in the higher-order moments, while those between Fig. 6 b and c, and between Fig. 7a and b are related to the turbulencescale condensation.

From the comparison above, it can be concluded that both the PDF and higher-order moments are important for a TOC model. Furthermore, it can be seen that the results from the FP 
TOC (Fig. 1c) combine the unrealistic features from Exps. 1 and 2. The higher-order moments seem to be more important because the cloud fraction and its vertical distribution from Exp. 2 are more similar to those from the FP TOC than those from the PP TOC, which suggests that the modification of the turbulence-scale condensation scheme alone does not improve the simulation much.

\section{A shallow to deep convective cloud transition case \\ 4.1 Design of experiment}

A shallow to deep convective cloud transition case is studied in this study. It is based on observations during the Large-Scale Biosphere-Atmosphere (LBA) experiment in Amazonia (Rondonia, Brazil) with additional measurements obtained during the TRMM-LBA (Tropical Rainfall Measuring Mission LBA ground validation program) on 23 February 1999. This study focuses on a part of the diurnal cycle from the sunrise to the early afternoon. The shallow convective clouds formed after a well-mixed convective boundary layer developed in the early morning. As the surface fluxes increased, the convective boundary layer deepened. The shallow cumuli then transformed to deep precipitating convective clouds around noon.

Working Group 4 of the GCSS (GEWEX Cloud System Study; GEWEX stands for Global Energy and Water Cycle Experiment) chose this case to study the transition from shallow to deep precipitating convection (Grabowski et al., 2005). The UCLA/LaRC CRM is one of the participating models in this study. Grabowski et al. found that single column models (SCMs) with convective parameterizations produce deep convection too soon, typically less than two hours after sunrise. Most CRMs with $1 \mathrm{~km}$ horizontal grid size are capable of capturing the qualitative aspects of the transition from shallow to deep precipitating convective clouds, but intermodel differences are significant for both 2-D and 3-D simulations.

The two-dimensional version of the UCLA/LaRC CRM with either the PP or FP TOCs is used to simulate this case, hereafter, the PP simulations and FP simulations. The only forcing 
comes from the increased surface fluxes and the evolving temperature tendencies due to radiative processes. Both are prescribed in order to reduce the complexities caused by the surface and radiative feedbacks. There is no large-scale advective tendencies of temperature and moisture. Details of the model configuration are the same as those described in Grabowski et al. (2005). Briefly, the horizontal domain size is $256 \mathrm{~km}$, with uniform grid size of $1 \mathrm{~km}$. The vertical grid size is $100 \mathrm{~m}$ near surface and stretches to $1.5 \mathrm{~km}$ at $30 \mathrm{~km}$.

Two ensembles of simulations were performed. One ensemble of simulations used the PP TOC, while the other used the FP TOC. Each ensemble consists of five simulations, whose initial conditions are slightly different from each other, but whose ensemble means are the same as in Grabowski et al. (2005). The reason for using the ensemble of simulations is to eliminate the random fluctuations caused by uncertainties in the initial condition.

\subsection{Temporal evolution}

Differences between the PP and FP simulations occur when the shallow cumuli begin to form and become larger in the deep convective phase. The time series of the surface precipitation rates for the two ensembles of simulations were plotted in Fig. 8 a. Most of the precipitation is due to convective clouds. The precipitation produced by the PP simulations is much less than that produced by the FP simulations and its onset occurs about 30 minutes later. The maximum precipitation rate from the PP simulations is about $20 \mathrm{~mm} \mathrm{day}^{-1}$ and is consistent with most of the other CRMs participating in the intercomparison project (Grabowski et al., 2005). The shallow cumuli occurred near hour 2 (Fig. 8b) for both CRMs, which is consistent with all of the CRM experiments that used a horizontal grid size of $100 \mathrm{~m}$ in Grabowski et al. (2005). Clouds with cloud top temperatures less than $245 \mathrm{~K}$ occur after hour 4 (Fig. $8 \mathrm{c}$ ). The cold cloud amount from the PP simulations is less than $10 \%$, while that from the FP simulations is as large as $25 \%$. The cold clouds, such as anvil or cirrus clouds, are usually associated with deep precipitating convective clouds. This suggests that there are more active deep convective clouds at the end of 
the FP simulations than at the end of the PP simulations. The center of the mass of the cloud fields (Fig. 8d) from the PP simulations is higher than that from the FP simulations before hour 4, related to deeper transitional convective clouds in the PP simulations as shown later. The total precipitable water increases gradually for both simulations before hour 3 (Fig. 8 e) because of the increased positive surface total water flux. However, the total precipitable water in the FP simulations decreases after hour 3.5 due to the large convective precipitation, while that for the PP simulations still keeps increasing due to the small convective precipitation. The highest cloud height increases with time (Fig. 8 f) when the shallow cumuli grow and some of them become deep convective clouds. The cloud height in the PP simulations before hour 3.5 is higher than that from the FP simulations, but the opposite is true when deep convective clouds develop. The magnitude of the difference in the cloud heights is larger in the deep convective phase than in the shallow cumulus phase. The liquid water path in both simulations (Fig. $8 \mathrm{~g}$ ) increases gradually after hour 2, but is in a quasi-steady state at the last hour, which is consistent with the benchmark simulations in Grabowski et al. (2005).

The development of deep convective clouds from shallow cumuli can be divided into three phases: shallow cumulus, transitional, and deep convective phases, based upon the time evolution of the cloud amount (Fig. 9). In the shallow cumulus phase, the cloud top is lower than $3 \mathrm{~km}$ and there are only shallow cumuli. This phase occurred before hour 3 for this case. In the transitional phase, the shallow cumuli grow and some of them become deep convective clouds. The domain-maximum cloud top height also increases from $3 \mathrm{~km}$ to $7 \mathrm{~km}$. This phase occurred between hour 3 and hour 5 in the PP simulations, but between hour 3.5 and hour 4.5 in the FP simulations. In the deep convective phase, the deep convective clouds develop. The clouds can reach the tropopause. This phase occurred in the last hour of the PP simulations and lasted slightly longer for the FP simulations. 
The cloud amount and its vertical structure from the PP simulations are greatly different from those of the FP simulations (Fig. 9). The maximum cloud amount from the FP simulations is comparable to the PP simulations in the shallow cumulus phase, but the cloud top is lower in the FP simulations. This feature was seen from the 1-D BOMEX simulation. This suggests that results from 1-D simulations are instructive to the more complex 2-D simulations. In the transitional phase, the transition from shallow cumuli to deep precipitating convective clouds occurs more rapidly in the FP simulations than in the PP simulations, accompanied by a rapid decrease of shallow cumulus population in the FP simulations. In the deep convective phase, an additional maximum center occurs in the upper troposphere in both simulations, but there are still many shallow cumuli present in the PP simulations.

In summary, the PP simulations produce more shallow cumuli in all the phases, and shallow cumulus clouds are deeper in the first two phases compared with the FP simulations. In the deep convective phase, more precipitation and cold clouds are produced in the FP simulations than in the PP simulations. The onset of the precipitation in the FP simulations is about 30 minutes earlier than in the PP simulations, due to a more rapid transition from shallow to deep convective clouds in the FP simulations. More detailed analyses will be presented below to understand the causes of these differences.

\subsection{Snapshots of cloud properties in different phases}

The cloud structure and evolution can be seen more clearly from the snapshots of cloud water in the three phases for representative simulations from the FP and PP ensembles (Fig. 10). In both simulations, the cloud width and depth increase with time. The PP simulation produces more shallow clouds in all three phases, whereas a large and deep convective cloud occupies the right half of the domain $(150 \mathrm{~km}$ to $200 \mathrm{~km})$ for the FP simulation. It can be further seen that the PP simulation also produces more narrower convective clouds than the FP 
simulation and that a spectrum of clouds with depths ranging from a couple of hundred meters to a couple of kilometers is present in the PP simulation. The continuous spectrum of clouds ensures a smooth transition from shallow cumuli to deep convective clouds because whenever a cloud type disappears, another cloud type grows and evolves into the type of previously dissipated clouds.

The cloud-scale motion from the FP simulation is much stronger than the PP simulation. This can be seen from the vertical velocity fields (Fig. 11). In the shallow cumulus phase (not shown), vertical velocity is always smaller than $0.5 \mathrm{~m} \mathrm{~s}^{-1}$ from the PP simulation, while there are two centers with vertical velocities greater than $0.5 \mathrm{~m} \mathrm{~s}^{-1}$ from the FP simulation. These magnitudes of vertical velocities are reasonable because shallow cumuli are not associated with strong updrafts. In the transitional phase, vertical velocities from the PP simulation exceed $0.5 \mathrm{~m}$ $\mathrm{s}^{-1}$ above $1 \mathrm{~km}$, while the maximum centers from the FP simulation are located at much higher levels. There are many grid columns with vertical velocities exceeding $0.5 \mathrm{~m} \mathrm{~s}^{-1}$ below $1 \mathrm{~km}$ in the FP simulation. This feature indicates that the resolved-scale transport below $1 \mathrm{~km}$ is more important in the FP simulation than in the PP simulation. In the deep convective phase, we can also see that vertical velocities are much larger in the FP simulation than in the PP simulation, due to stronger organization of convection (Fig. 10f).

The FP simulation produces cold pools that are stronger and occur earlier than those in the PP simulation. This can be seen from the perturbation field of potential temperature (Fig. 12). In the transitional phase, the PP simulation did not produce large potential temperature perturbations, whereas the FP simulation produced large areas of negative temperature perturbations with magnitudes less than $-0.5 \mathrm{~K}$ below $3 \mathrm{~km}$. Because the cold air inhibits the subgrid scale and grid-scale upward sensible heat and moisture transport, and is detrimental to the development of shallow cumuli, the population of shallow cumuli decreases drastically in both 
the FP and PP simulations (Fig. 10e and f) when the cold pools are widespread in the deep convective phase.

In summary, the differences in the strengths of the cold pools and cloud-scale circulations can explain the differences in the cloud distributions between the FP and PP simulations discussed earlier, especially those in the transitional and deep convective phases.

\subsection{Mean state and fluxes}

The domain-averaged potential temperature and the water vapor mixing ratio have smaller vertical gradients in the PP simulations than in the FP simulations (Fig. 13). They are warmer and drier below $1 \mathrm{~km}$ but colder and moister above $1 \mathrm{~km}$ in the PP simulations than in the FP simulations, respectively. Guichard et al. (2005) found that the main difference between CRMs and SCMs in the simulations of diurnal cycle of convection is that CRMs tend to moist and warm the free troposphere gradually, but the adjustments of SCMs are too quick. Fig. 13 further shows that the degree of moistening and warming in CRMs is highly dependent on the subgridscale parameterizations of turbulence.

The differences in the domain-averaged states suggest that there are some differences in the convective instability. The time series of the convective available potential energy (CAPE) is shown in Fig. 14. The CAPE is calculated by CAPE $=\int_{z_{0}}^{z_{1}}\left[g\left(\theta_{c}-\theta_{e n v}\right) / \theta_{e n v}\right] d z$, where $z_{0}$ is the level of free convection (LFC), $z_{1}$ is the level of neutral buoyancy (LNB), $g$ is the acceleration of gravity, $\theta_{c}$ is the potential temperature of an air parcel lifted from the surface up to the LFC, and $\theta_{\text {env }}$ is the potential temperature of the environment. From Fig. 14, we can see that the CAPE from the FP simulations is larger than that from the PP simulations before the deep convective phase. The two ensembles of simulations also show a gradual increase in CAPE over the first 2 hours due to the increase of surface sensible and latent heat fluxes and the radiative 
cooling. When the shallow cumuli develop, the CAPE stops increasing. It is in a quasi-steady state for the deep convective phase of the PP simulations, but it tends to decrease in the deep convective phase of the FP simulations due to the stronger stabilization effect of deep convection in the FP simulations.

The differences in the domain-averaged profiles of the boundary-layer potential temperature and water vapor between the FP and PP simulations are closely related to the turbulent and resolved-scale transports. The total liquid-water potential temperature and total water flux (Fig. 15) from the FP simulations do not reach a level as high as that in the PP simulations in the shallow cumulus phase. This is consistent with the $1 \mathrm{D}$ simulation of the BOMEX cumulus case, i.e., less effective turbulent transport in the FP simulations. In all the phases, turbulent transports in the PP simulations are much larger than those in the FP simulations. The turbulent transports are also much larger than the resolved-scale transports in the shallow cumulus and transitional phases of the PP simulations, which enhances the vertical mixing of the mean profiles and inhibits the increase of the CAPE.

\subsection{Interactions between shallow cumuli and deep convective clouds}

We have compared the time series, the cloud properties, the mean thermodynamic profiles and the resolved-scale and turbulent transports from the FP and the PP simulations. Cumulus clouds grow gradually and become deep convective clouds with a maximum height of $12-14 \mathrm{~km}$ in both sets of simulations. However, there are a lot of differences between the two simulations.

The PP simulations have larger turbulent transports. The potential temperature and water vapor mixing ratio are more well mixed than in the FP simulations. The CAPE is smaller until near the end of the simulations. More and deeper shallow cumuli are produced. The deep convection in the PP simulations is delayed due to the stabilization effects of the shallow cumuli and the turbulent mixing. The productions of precipitation and cold pools are also delayed. In the FP simulations, the potential temperature and water vapor mixing ratio are less well mixed and 
fewer shallow cumuli are produced. The production of the precipitation occurs earlier and the cold pools form earlier. The cold pools are usually located below $3 \mathrm{~km}$, which is detrimental to the vertical transport of the sensible heat and the total water. Shallow cumuli can only be produced near the edge of the cold pools where the turbulent transport is not prohibited in the FP simulations.

The stronger turbulent mixing associated with the shallow cumuli basically slows down the increase of CAPE and inhibits the early growth of the deep convective clouds in the transitional phases. When the deep convective clouds fully develop and precipitation is produced, the cold pools produced by the evaporation of the precipitation are not favorable to shallow cumuli, except near the edges of the cold pools.

\section{Summary and discussion}

The abilities of the double-Gaussian based and the single-Gaussian based TOC models to simulate the shallow cumuli and their transition to deep convective clouds are compared in this study. In the double-Gaussian based TOC, we use the analytical double-Gaussian II proposed by Larson et al. (2002). Only the third-order moments of the liquid water potential temperature, the total water, and the vertical velocity are predicted to determine the double-Gaussian PDF. So this model is called the PP model. The single-Gaussian based TOC is called the FP model since all the third-order moments are predicted.

BOMEX, a "pure" shallow cumuli case, was simulated by the single-column versions of the FP and PP models. The PP model improves the simulation of shallow cumuli greatly over the FP model by producing more realistic cloud structures in both the magnitude of cloud fraction and the location of cloud top. Large differences between the second- and third-order moments produced by the FP and PP simulations appear in the cloud layer. Third-order moments of vertical velocity, liquid-water potential temperature and total water mixing ratio from the FP model are almost zero, while the second-order moments from the FP model have their peak values at lower levels. These differences are due to various reasons. Further experiments and analytical studies 
have shown that both the PDF based turbulence-scale condensation and higher-order moments are important to realistic simulations of the boundary-layer shallow cumuli. The single-Gaussian PDF based turbulence-scale condensation predicts a lower cloud top, while the higher-order moments from the quasi-Gaussian approximation produces very large cloud faction and an unrealistic cloud fraction profile.

A shallow to deep convective cloud transition case, based on observations during the LBA experiment in Amazonia with specific data collected during the TRMM-LBA on 23 February 1999, was also simulated by the 2D versions of the FP and PP models. Shallow convective clouds transformed to deep precipitating convective clouds as the surface fluxes increased after the sunrise. The only forcing comes from the prescribed surface fluxes and radiative effects. There are no large-scale advective tendencies of temperature and moisture. These simulations have been analyzed in greater detail than those featured in the intercomparison study of Grabowski et al. (2005).

Both the FP and PP simulations can capture qualitatively the transition from the shallow cumuli to deep convective clouds. However, the adjustment processes are much different. There is more precipitation and cold cloud produced in the FP simulations than in the PP simulations. The onset of the precipitation of the FP simulations is about 30 minutes earlier than that of the PP simulations. In the first two phases, the PP simulations produce more and deeper shallow cumuli than the FP simulations, but the FP simulations produce larger and wider convective clouds in the deep convective phase than the PP simulations. The temporal evolution of cloud and precipitation is found to be closely related to the turbulent transport, the cold pool and the cloud-scale circulation.

The PP simulations have larger turbulent transports. The potential temperature and water vapor mixing ratio are more well mixed than in the FP simulations. More and deeper shallow cumuli are produced. The deep convection is delayed due to the stabilization effects of the shallow cumuli and the turbulent mixing. The production of the precipitation and the cold pool is 
also delayed. In the FP simulations, the turbulent transport is much smaller. The potential temperature and water vapor mixing ratio are less well mixed and fewer shallow cumuli are produced. Both the production of the precipitation and the formation of cold pools occur earlier. The cold pools are usually located below $3 \mathrm{~km}$, which is detrimental to the vertical transport of the sensible heat and the total water. Shallow cumuli can only be produced near the edges of cold pools in the FP simulations where the turbulent transport is not prohibited.

The large turbulent mixing associated with the shallow cumuli slows down the increase of the CAPE and inhibits the early growth of the deep convective clouds in the transitional phase. When the deep convective clouds fully develop and the precipitation is produced, the cold pools produced by the evaporation of the precipitation are not favorable to shallow cumuli.

Petch et al. (2002) studied the impact of horizontal resolution on the simulations of convective clouds. They reported that the precipitation, the potential temperature and total water fluxes are sensitive to the horizontal resolution. A sophisticated subgrid-scale scheme can reduce this sensitivity. We found that the differences caused by the resolution are much smaller than those caused by the different TOC schemes (not shown). This may be due to the fact that both TOC schemes can simulate the convective clouds and suggests the importance of sophisticated subgrid-scale schemes in reducing the sensitivity of simulations to the resolution.

This study may shed some light on the shallow and deep convection parameterization in general circulation models. The turbulent transport is very important for the evolution of the deep convective clouds. Proper turbulent transports ensure the smooth transition from shallow cumuli to deep convective clouds.

Acknowledgments: This research was partially supported by the Environmental Sciences Division of the U.S. Department of Energy as part of the Atmospheric Radiation Measurement Program, under interagency agreement DE-AI02-02ER63318 to NASA Langley Research Center. Dr. Z. A. Eitzen is thanked for improving the writing of this manuscript. Thanks also go to Drs. 
Bjorn Stevens and Pier Siebesma for providing LES data from the intercomparison workshop to test our 1-D TOC model. Special thanks also go to Dr. Bjorn Stevens who provided the UCLALES. 


\section{References}

Albrecht, B. A., 1979: A model of the thermodynamic structure of the trade-wind boundary-layer. Part II. Applications. J. Atmos. Sci., 36, 90-98.

André, J. C., G. De Moor, P. Lacarrère, and R. Du Vachat, 1976a: Turbulence approximation for inhomogeneous flows. Part I: The clipping approximation. J. Atmos. Sci., 33, 476-481.

$-, \longrightarrow,-, 1976 \mathrm{~b}$ : Turbulence approximation for inhomogeneous flows. Part II: The numerical simulation of a penetrative convection experiment. J. Atmos. Sci., 33, 482-491.

Arakawa, A., 1969: Parameterization of cumulus convection. Proc. WMO/IUGG Symp. Numerical Weather Prediction, Tokyo, Japan, Japan Meteorological Agency, 1-6.

Betts, A., 1973: Non-precipitating cumulus convection and its parameterization. Quart. J. Roy. Meteor. Soc., 99, 178-196.

Bougeault, P., 1981a: Modeling the trade-wind cumulus boundary layer. Part I: Testing the ensemble cloud relations against numerical data. J. Atmos. Sci., 38, 2414-2428.

Bougeault, P., 1981b: Modeling the trade-wind cumulus boundary layer. Part II: A higher-order one-dimensional model. J. Atmos. Sci., 38, 2429-2439.

Cheng A., K.-M. Xu, and J.-C. Golaz, 2004: Liquid water oscillation in modeling boundary-layer cumuli with third-order turbulence closure models. J. Atmos. Sci., 61, 1621-1629.

Clark, R. H., A. J. Dyer, R. R. Brook, D. G. Reid, and A. J. Troup, 1971: The Wangara experiment: Boundary layer data. Tech. Paper 19, Division Meteor. Phys., CSIRO, 
Australia.

Golaz, J.-C., V. E. Larson, and W. R. Cotton, 2002a: A PDF-based model for boundary layer clouds. Part I: Method and model description. J. Atmos. Sci., 59, 3540-3551.

$\longrightarrow,-$, and $\longrightarrow, 2002 \mathrm{~b}$ : A PDF-based model for boundary layer clouds. Part II: Model results. J. Atmos. Sci., 59, 3552-3571.

Grabowski, W. W., and coauthors, 2005: Daytime convective development over land: a model intercomparison based on LBA observations. Submitted to Quart. J. Roy. Meteor. Soc.

Guichard, F., and coauthors, 2005: Modelling the diurnal cycle of deep precipitating convection over land with CRMs and SCMs. Quart. J. Roy. Meteor. Soc., 130, 3139-3172.

Krueger, S. K., 1988: Numerical simulation of tropical cumulus clouds and their interaction with the subcloud layer. J. Atmos. Sci., 45, 2221-2250.

Krueger, S. K., and A. Bergeron, 1994: Modeling the trade cumulus boundary layer. Atmos. Res., 33, 169-192.

Lappen, C.-L., and D. A. Randall, 2001a: Toward a unified parameterization of the boundary layer and moist convection. Part I: A new type of mass-flux model. J. Atmos. Sci., 58, 2021-2036.

Lappen, C.-L., and D. A. Randall, 2001b: Toward a unified parameterization of the boundary layer and moist convection. Part III: Simulations of clear and cloudy convection. J. Atmos. Sci., 58, 2052-2072.

Larson, V. E., J.-C. Golaz, and W. R. Cotton, 2002: Small-scale and mesoscale variability in cloudy boundary layers: Joint probability density functions. J. Atmos. Sci., 59, 3519-3539.

Lewellen, W. S., and S. Yoh, 1993: Binormal model of ensemble partial cloudiness. J. Atmos. Sci., 
50, 1228-1237.

Mellor, G. L., 1977: The Gaussian cloud model relations. J. Atmos. Sci., 34, 344-355.

Petch, J. C., Brown, A. R. and Gray, M. E. B., 2002: The impact of horizontal resolution on convective development in simulations of the diurnal cycle over land. Quart. J. Roy. Meteor. Soc., 128, 2031-2044.

Randall, D. A., 1987: Turbulent fluxes of liquid water and buoyancy in partly cloudy layers. $J$. Atmos. Sci., 44, 850-858.

Siebesma, A. P., C. S. Bretherton, A. Brown, A. Chlond, J. Cuxart, P. G. Duynkerke, H. Jiang, M. Khairoutdinov, D. Lewellen, C.-H. Moeng, E. Sanchez, B. Stevens, and D. E. Stevens, 2003: A large-eddy simulation intercomparison study of shallow cumulus convection. $J$. Atmos. Sci. 60, 1201-1219.

Sommeria, G., and J. W. Deardorff, 1977: turbulence-scale condensation in models of nonprecipitating clouds. J. Atmos. Sci., 34, 344-355.

Stevens, B., C.-H. Moeng, and P. P. Sullivan, 1999: Large-eddy simulations of radiatively driven convection: Sensitivities to the representation of small scales. J. Atmos. Sci., 56, 39633984.

$\mathrm{Xu}$, K.-M., and D. A. Randall, 1995: Impact of interactive radiative transfer on the macroscopic behavior of cumulus ensembles. Part I: Radiation parameterization and sensitivity tests. $J$. Atmos. Sci., 52, 785-799. 


\section{Figure Captions}

Figure 1: Time series of (a) UCLA LES-, (b) PP-, and (c) FP-simulated cloud fraction for the BOMEX case.

Figure 2: Profiles of (a) $\overline{w^{\prime} w^{\prime}}$, (b) $\overline{\theta_{l}^{\prime 2}}$, (c) $\overline{q_{t}^{\prime 2}}$, and (d) $\overline{q_{t}^{\prime} \theta_{l}^{\prime}}$ averaged between hours 3 and 5 of the BOMEX simulations. The solid line denotes the UCLA-LES, the dotted line denotes the PP model, the dashed line denotes the FP model, and the shaded area [in (a) only] has a width of twice the standard deviation around the mean profile calculated from LESs (please see Siebesma et al. 2003 for details of the LESs).

Figure 3: Profiles of (a) $\rho L_{v} \overline{w^{\prime} q_{t}^{\prime}}$, and (b) $\rho c_{p} \overline{w^{\prime} \theta_{l}^{\prime}}$ averaged between hours 3 and 5 of the BOMEX simulations, where $\rho$ is the density of the air, $L_{v}$ is the latent heat of evaporation and $c_{p}$ is the specific heat capacity. The solid line denotes the UCLA-LES, the dotted line denotes the PP model, the dashed line denotes the FP model, and the shaded area has a width of twice the standard deviation around the mean profile calculated from LESs.

Figure 4: Profiles of (a) $\overline{w^{\prime 3}}$, (b) $\overline{\theta_{l}^{\prime 3}}$, and (c) $\overline{q_{t}^{\prime 3}}$ averaged between hours 3 and 5 of the BOMEX simulations. The solid line denotes the UCLA-LES, the dotted line denotes the PP model, the dashed line denotes the FP model, and the shaded area has a width of twice the standard deviation around the mean profile calculated from LESs.

Figure 5: Time series of cloud fraction for (a) EXP. 1, and (b) EXP. 2. See text for details.

Figure 6: Joint PDFs of liquid-water potential temperature and total water mixing ratio at $z=780$ $m$ between hours 4 and 5 of the BOMEX case produced by (a) the UCLA-LES, (b) the double-Gaussian PDF and (c) the single-Gaussian PDF. The moments used to determine the PDFs are diagnosed from the UCLA-LES. The straight line is the saturation curve. 
Figure 7: Joint PDFs of liquid-water potential temperature and total water mixing ratio at $z=780$ $\mathrm{m}$ between hours 4 and 5 of the BOMEX case produced by (a) the double-Gaussian PDF and (b) the single-Gaussian PDF. The moments used to determine the PDFs are predicted from the FP simulations. The straight line is the saturation curve.

Figure 8: Time series of (a) surface precipitation, (b) total cloud fraction, (c) cold cloud fraction, (d) height of the center of mass of the cloud field, (e) precipitable water, (f) maximum cloud top height, and (g) cloud liquid water path for the shallow cumuli to deep convective clouds transition case. The solid line denotes the ensemble mean of the five PP simulations, and the dotted line denotes the FP simulations.

Figure 9: Time-height cross-sections of cloud fraction for (a) PP simulations, and (b) FP simulations.

Figure 10: Snapshots of liquid water mixing ratio $\left(10^{-5} \mathrm{~kg} \mathrm{~kg}^{-1}\right)$ for (a) a PP simulation at hour 2.5, (b) an FP simulation at hour 2.5, (c) a PP simulations at hour 4, (d) an FP simulations at hour 4, (e) a PP simulations at hour 6, and (f) an FP simulations at hour 6.

Figure 11: Snapshots of vertical velocity $\left(\mathrm{m} \mathrm{s}^{-1}\right)$ for (a) a PP simulation at hour 4, (b) an FP simulation at hour 4, (c) a PP simulation at hour 6, and (d) an FP simulation at hour 6.

Figure 12: Snapshots of perturbation potential temperature (K) for (a) a PP simulation at hour 4, (b) an FP simulation at hour 4, (c) a PP simulation at hour 6, and (d) an FP simulation at hour 6.

Figure 13: Domain-averaged profiles of the potential temperature (a) at hour 2.5, (b) at hour 4, and (c) at hour 6, and the water vapor mixing ratio (d) at hour 2.5 , (e) at hour 4 , and (f) at hour 6. The solid line denotes the PP simulations, and the dotted line denotes the FP simulations.

Figure 14: Time series of the CAPE for the FP simulations (dotted line) and the PP simulations (solid line). 
Figure 15: Domain-averaged transports of the liquid water potential temperature (a) at hour 2.5, (b) at hour 4, and (c) at hour 6, and the total water (d) at hour 2.5, (e) at hour 4, and (f) at hour 6. The solid line denotes the total transport of the PP simulations, the dashed line denotes the turbulent transport of the PP simulations, the dotted line denotes the total transport of the FP simulations, and the dot-dashed line denotes the turbulent transport of the FP simulations. 
Figure 1
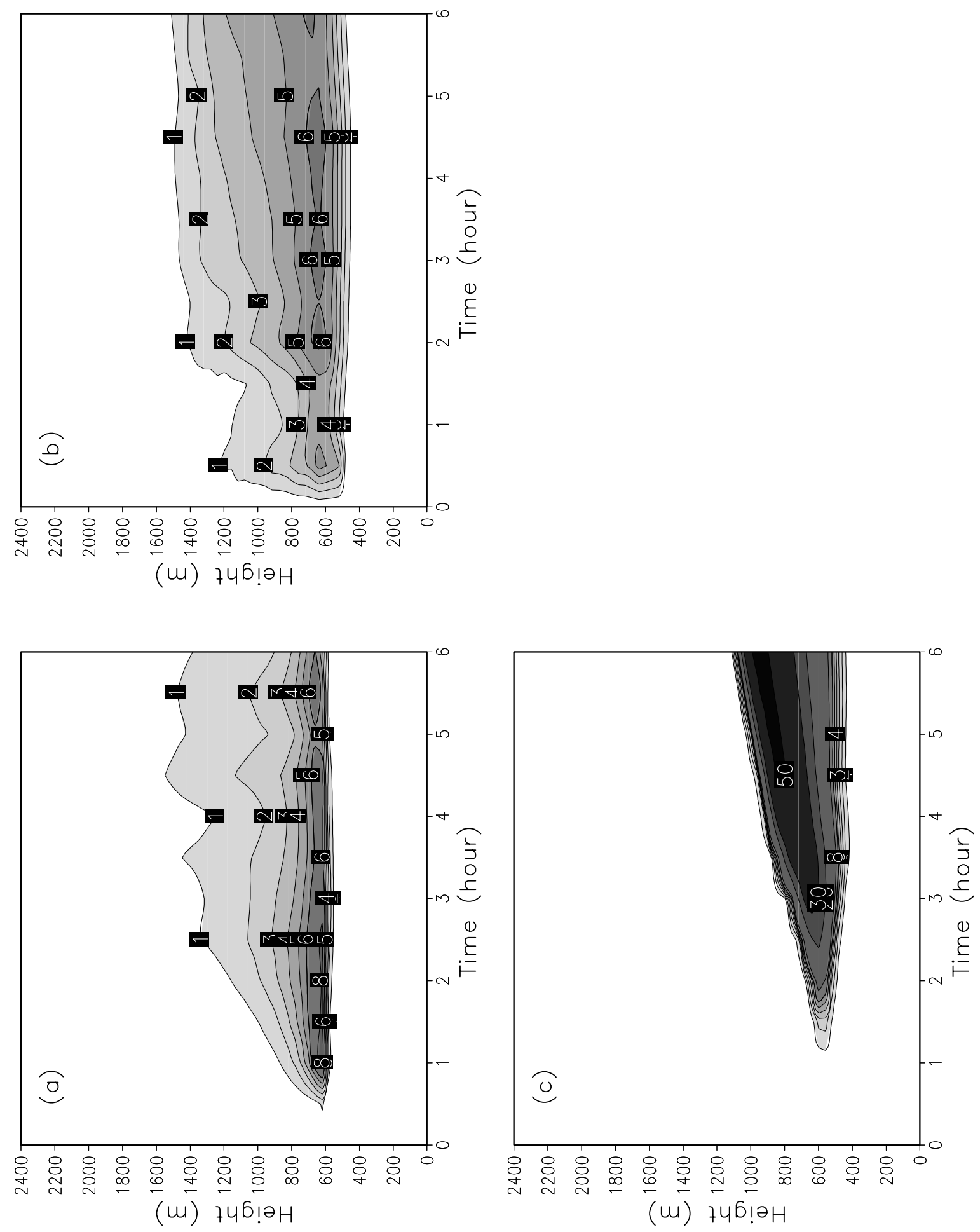
Figure 2
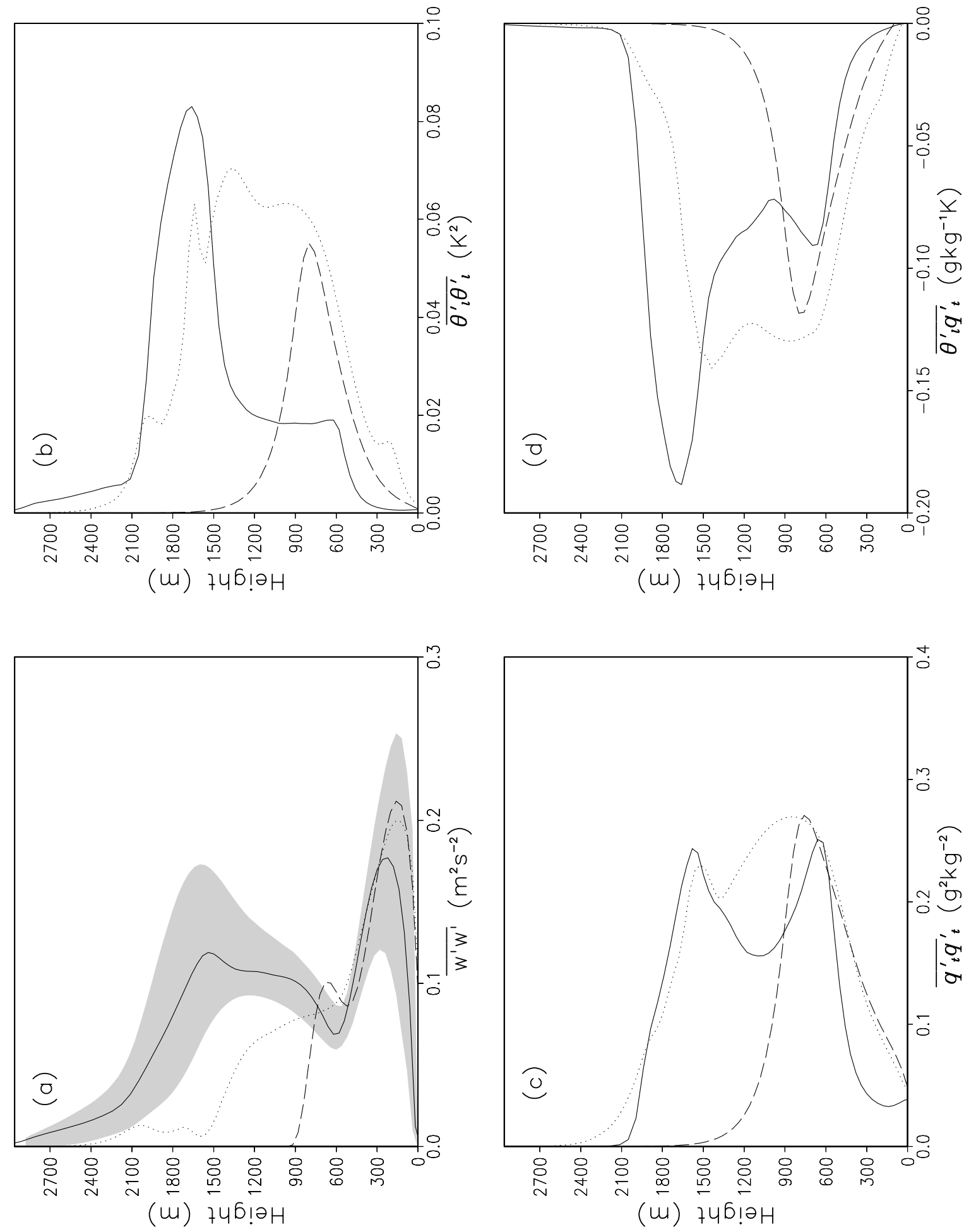
Figure 3
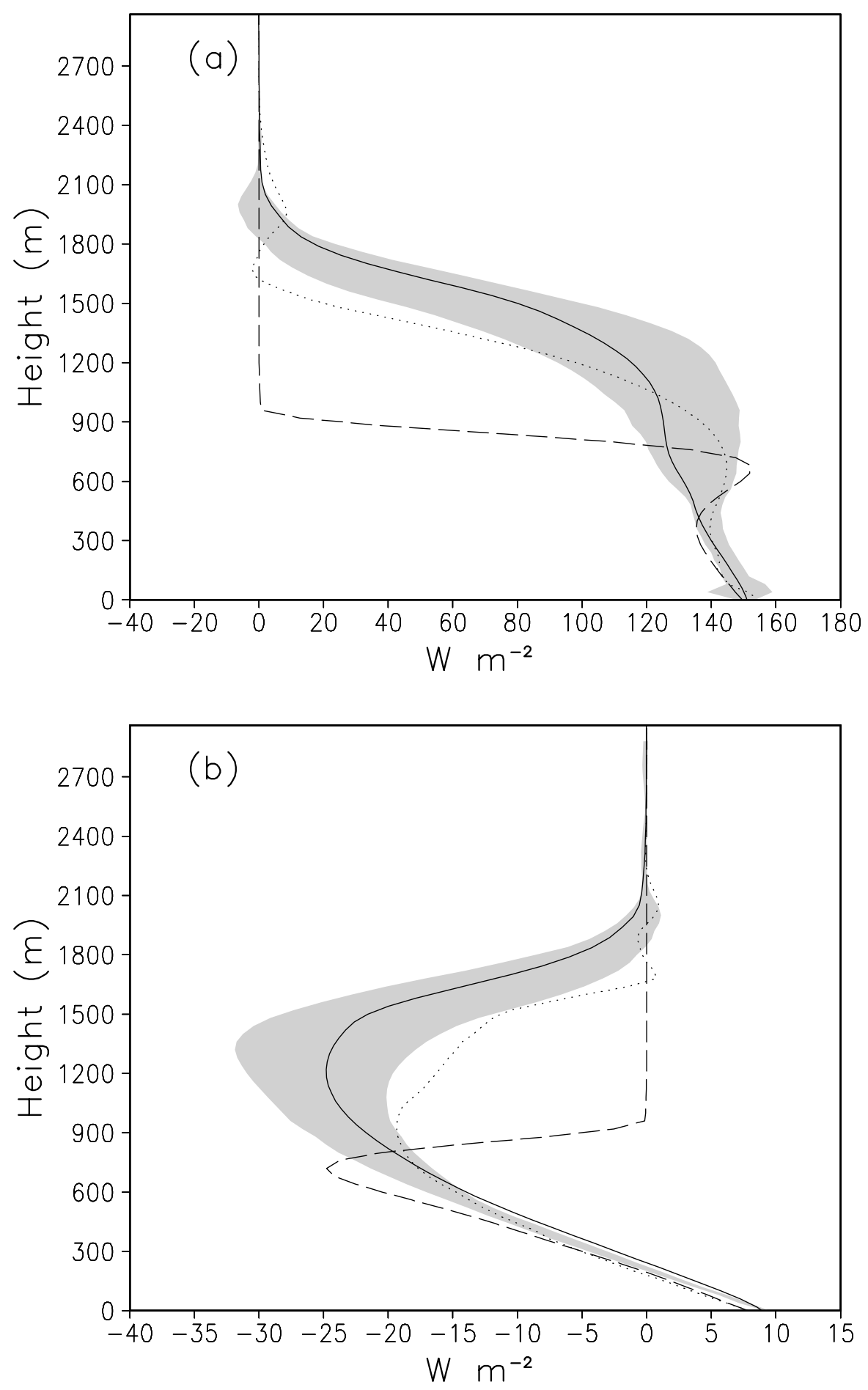
Figure 4
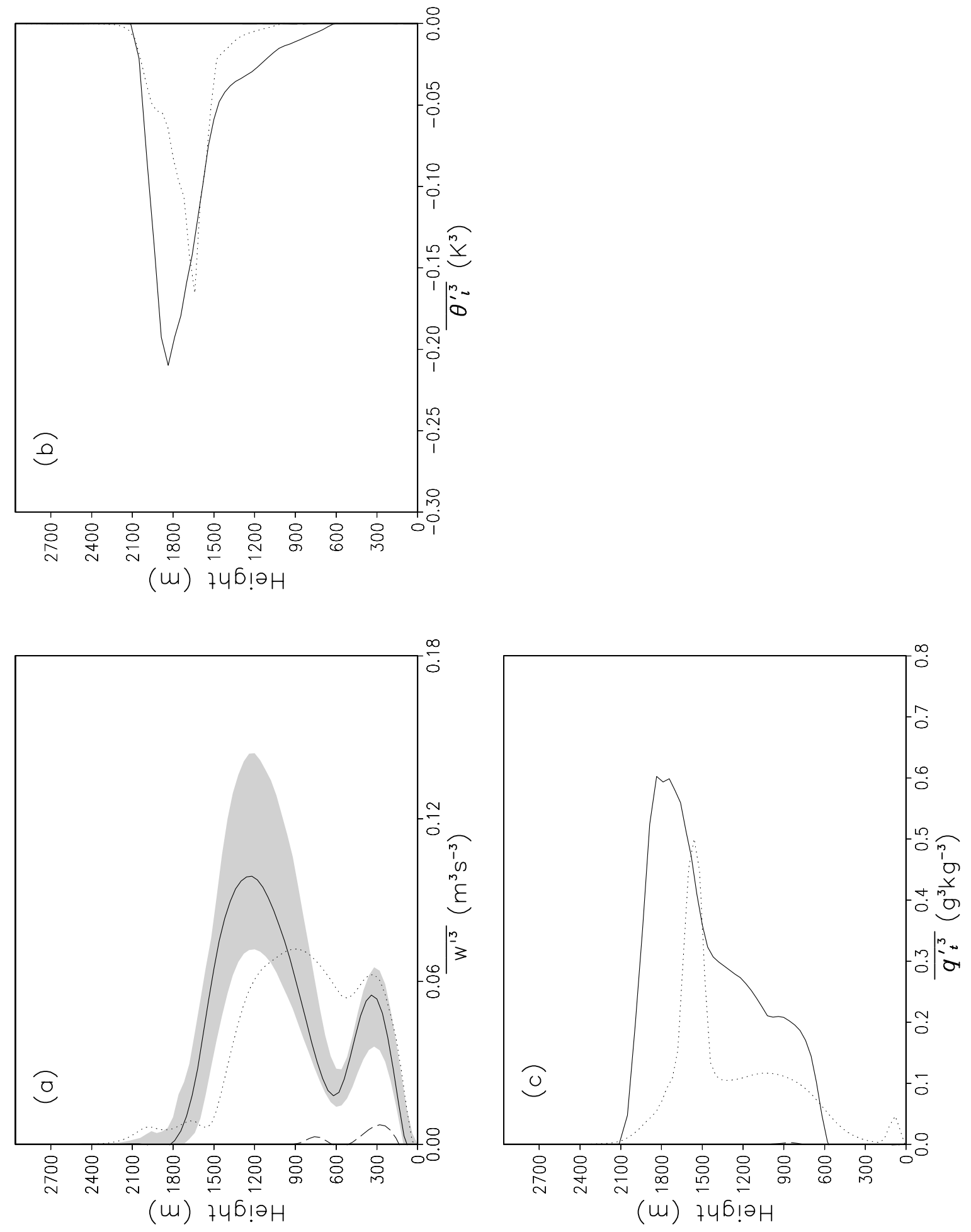
Figure 5
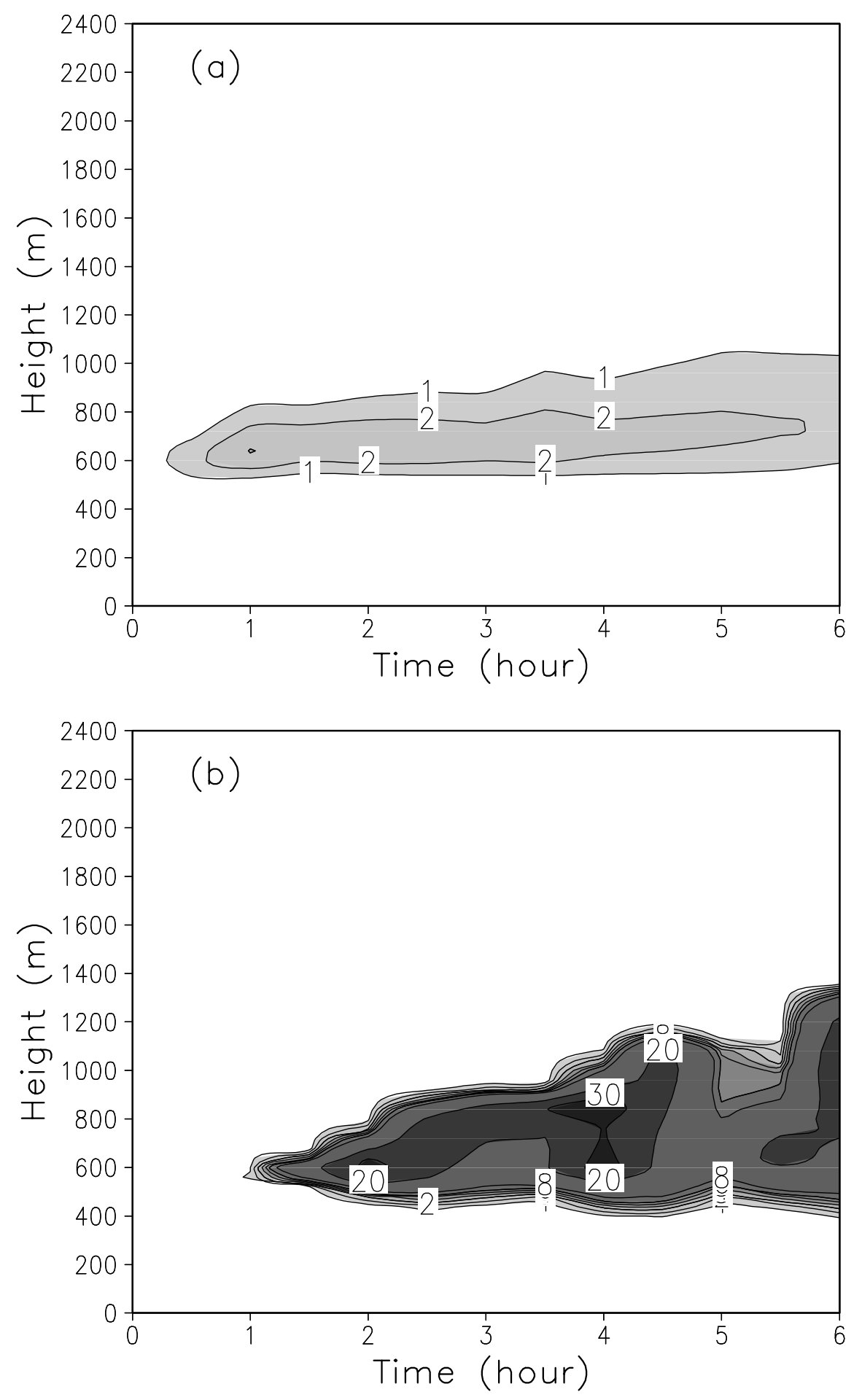
Figure 6
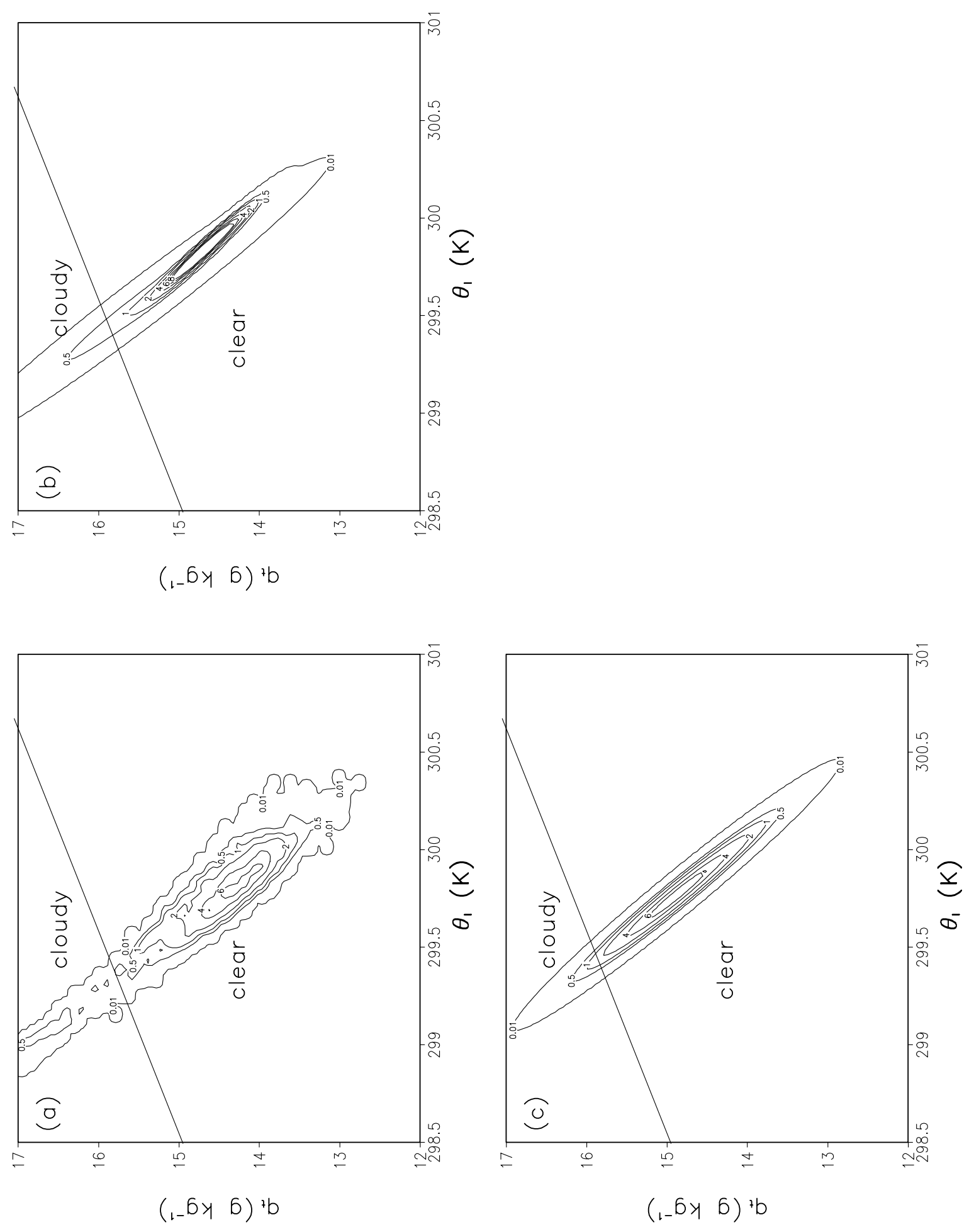
Figure 7
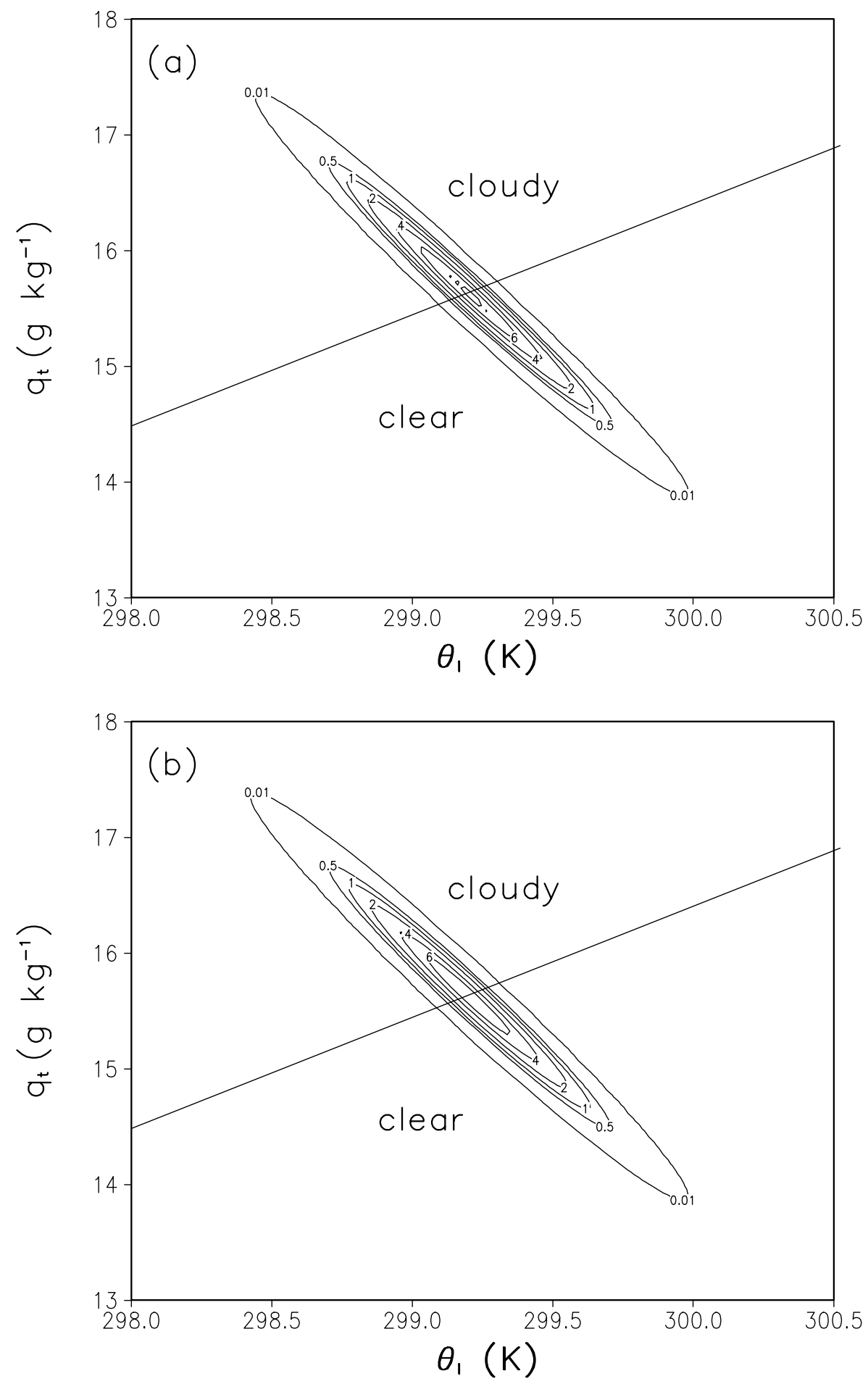
Figure 8

Figure 8 (continued)
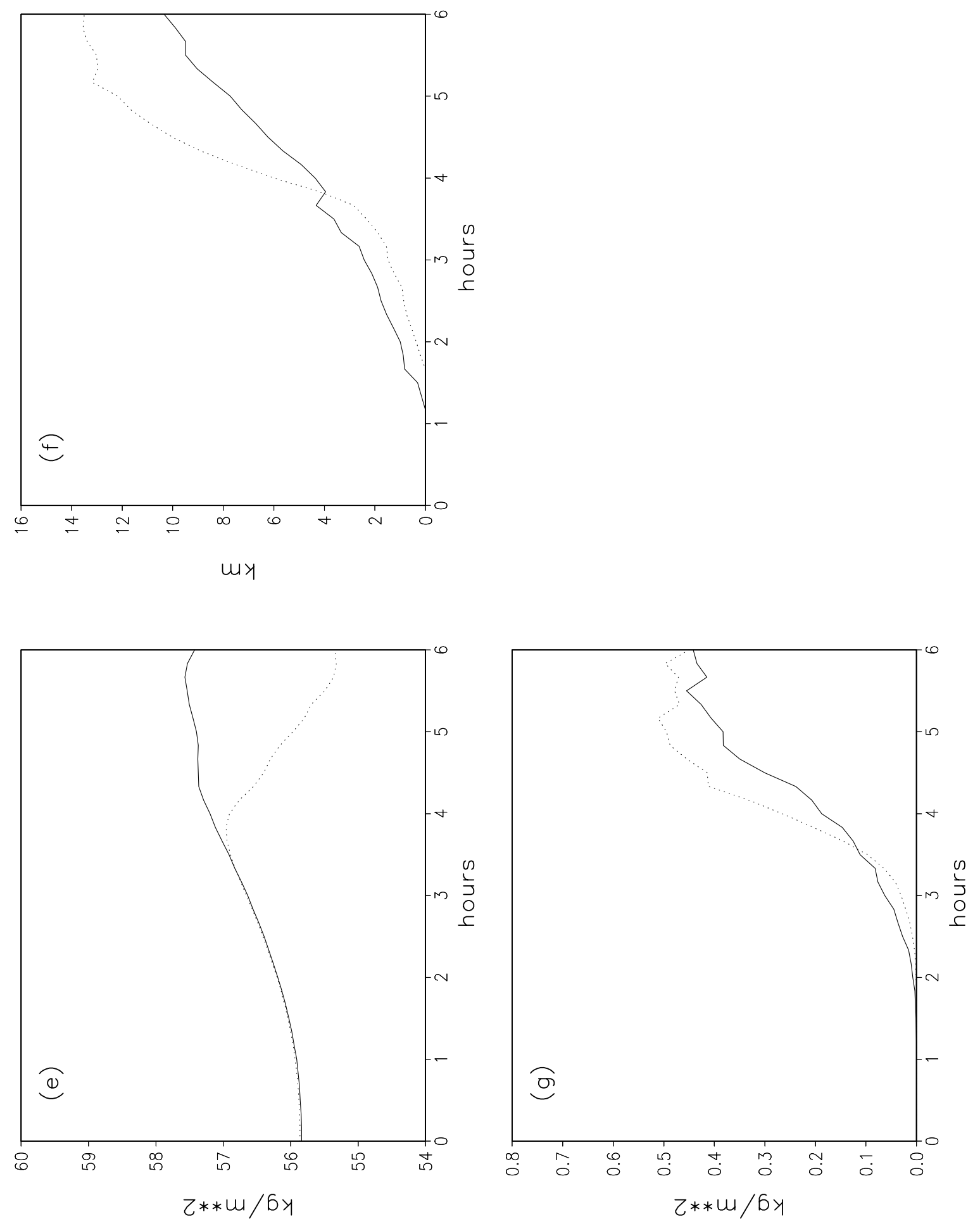
Figure 9
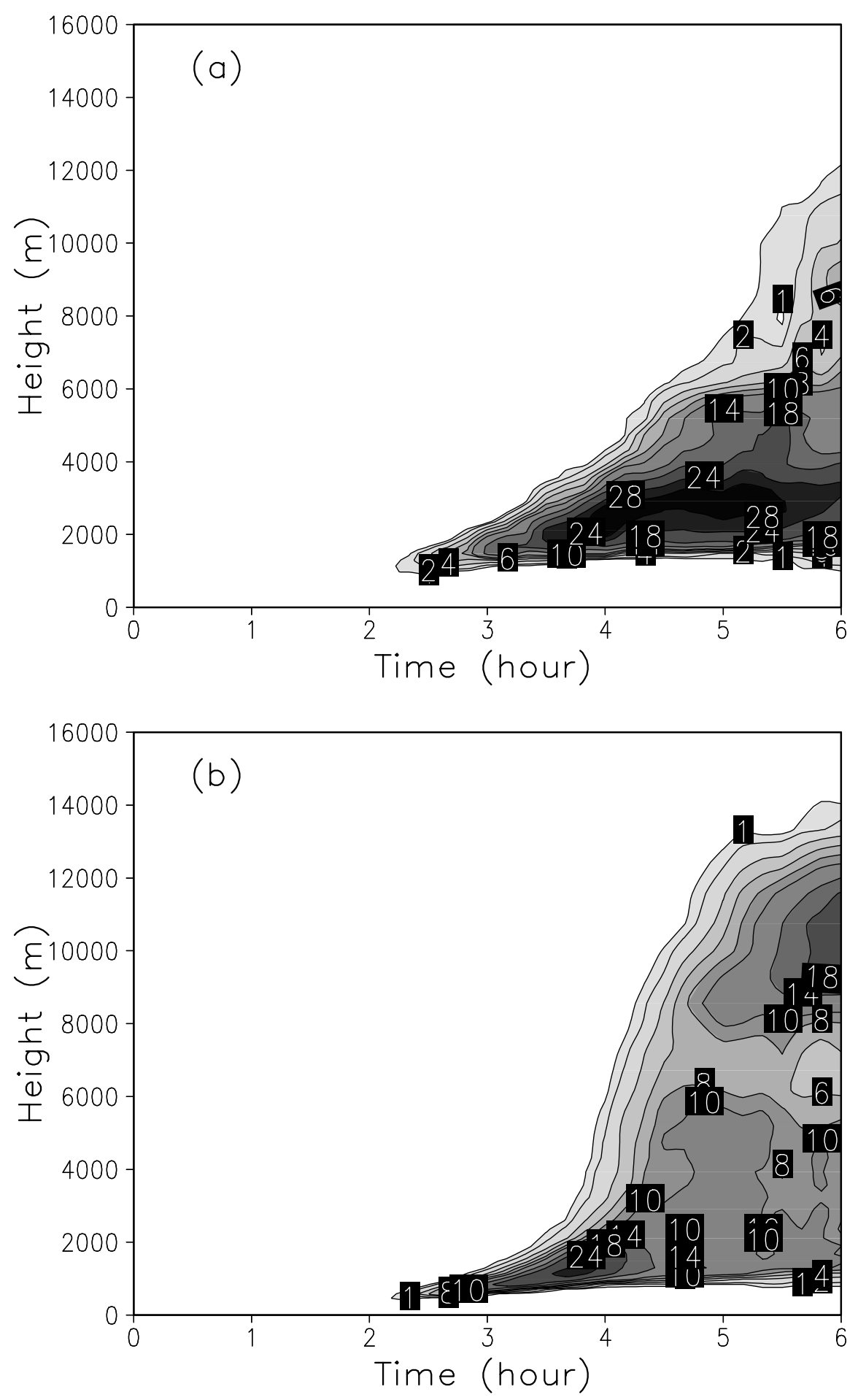
Figure 10
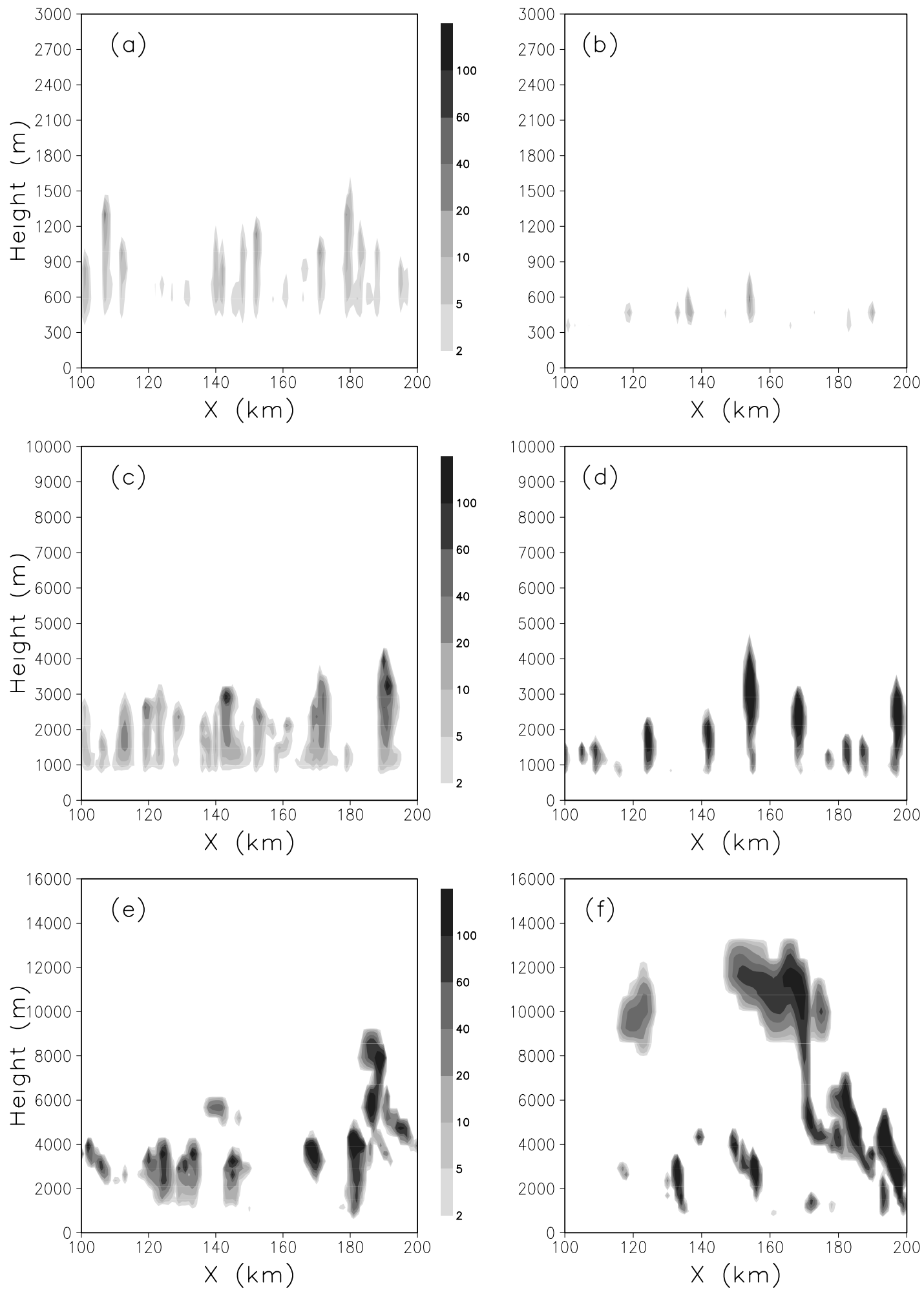
Figure 11
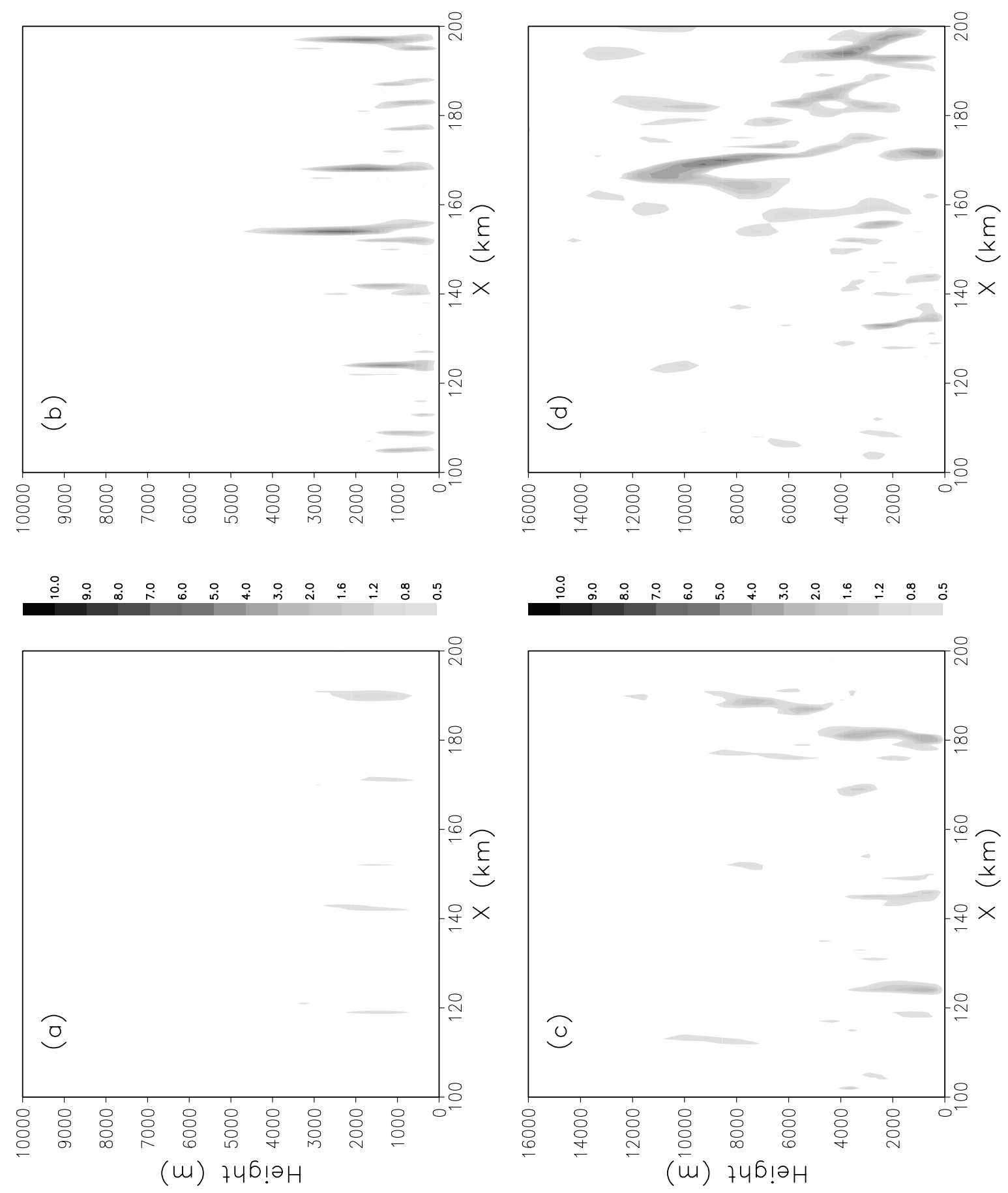
Figure 12
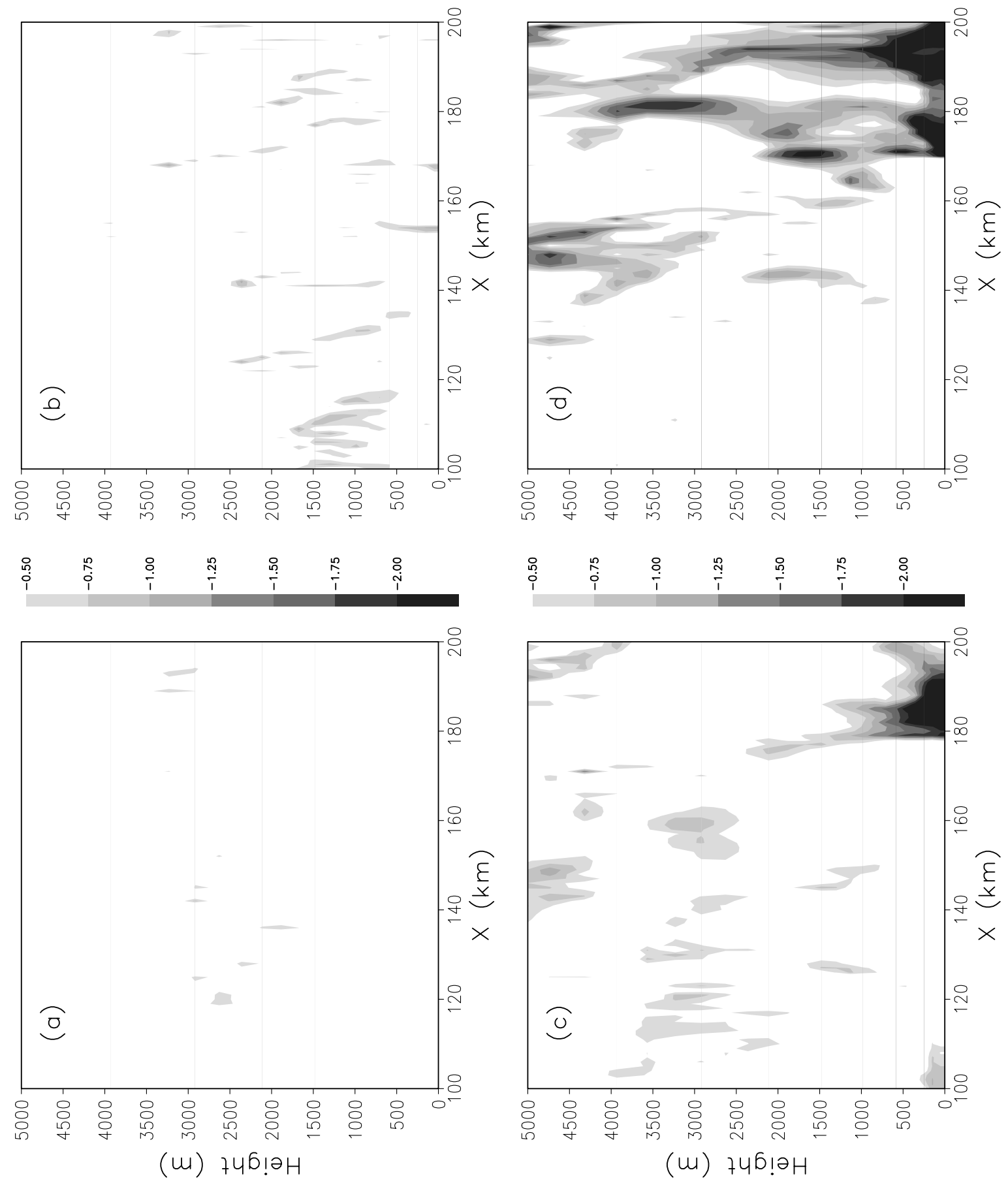
Figure 13
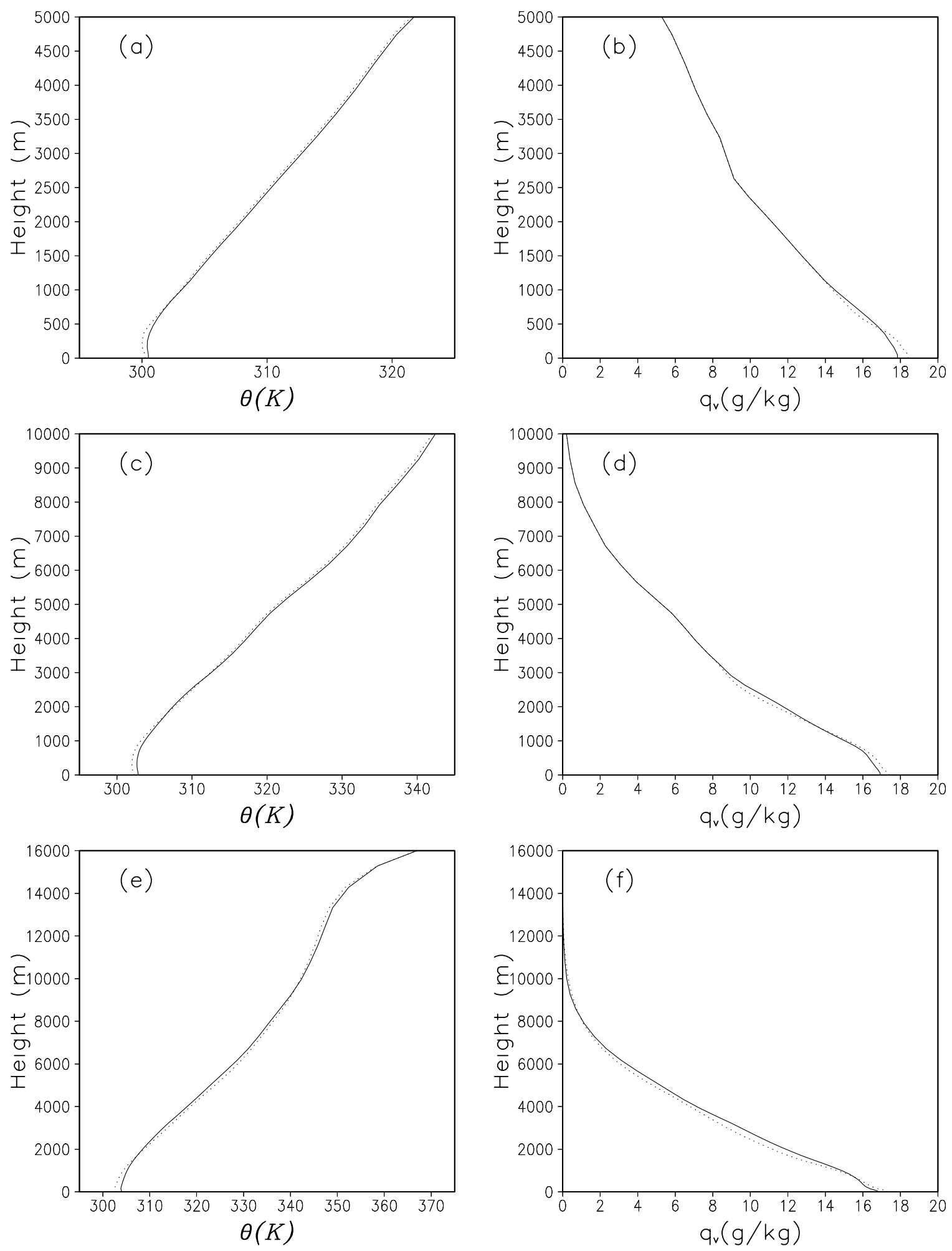
Figure 14

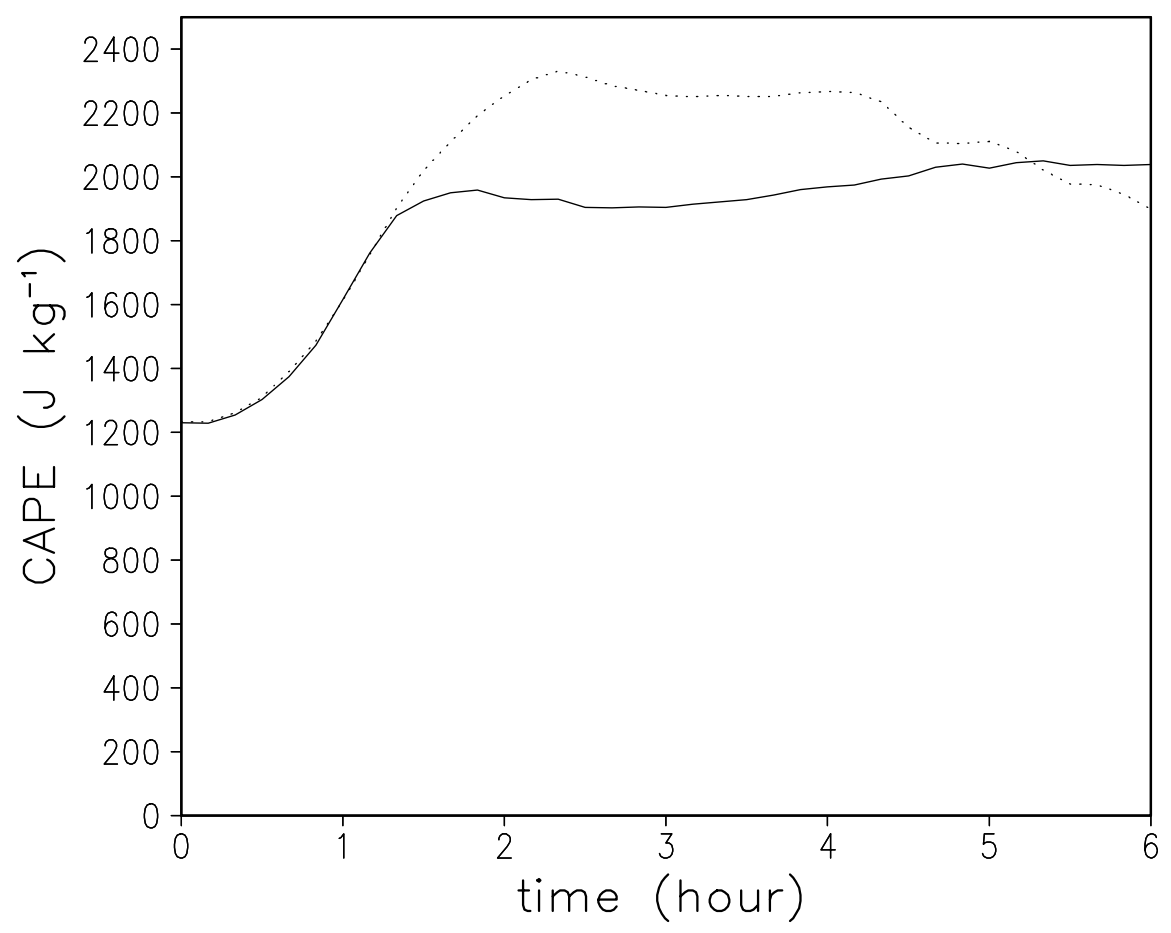


Figure 15
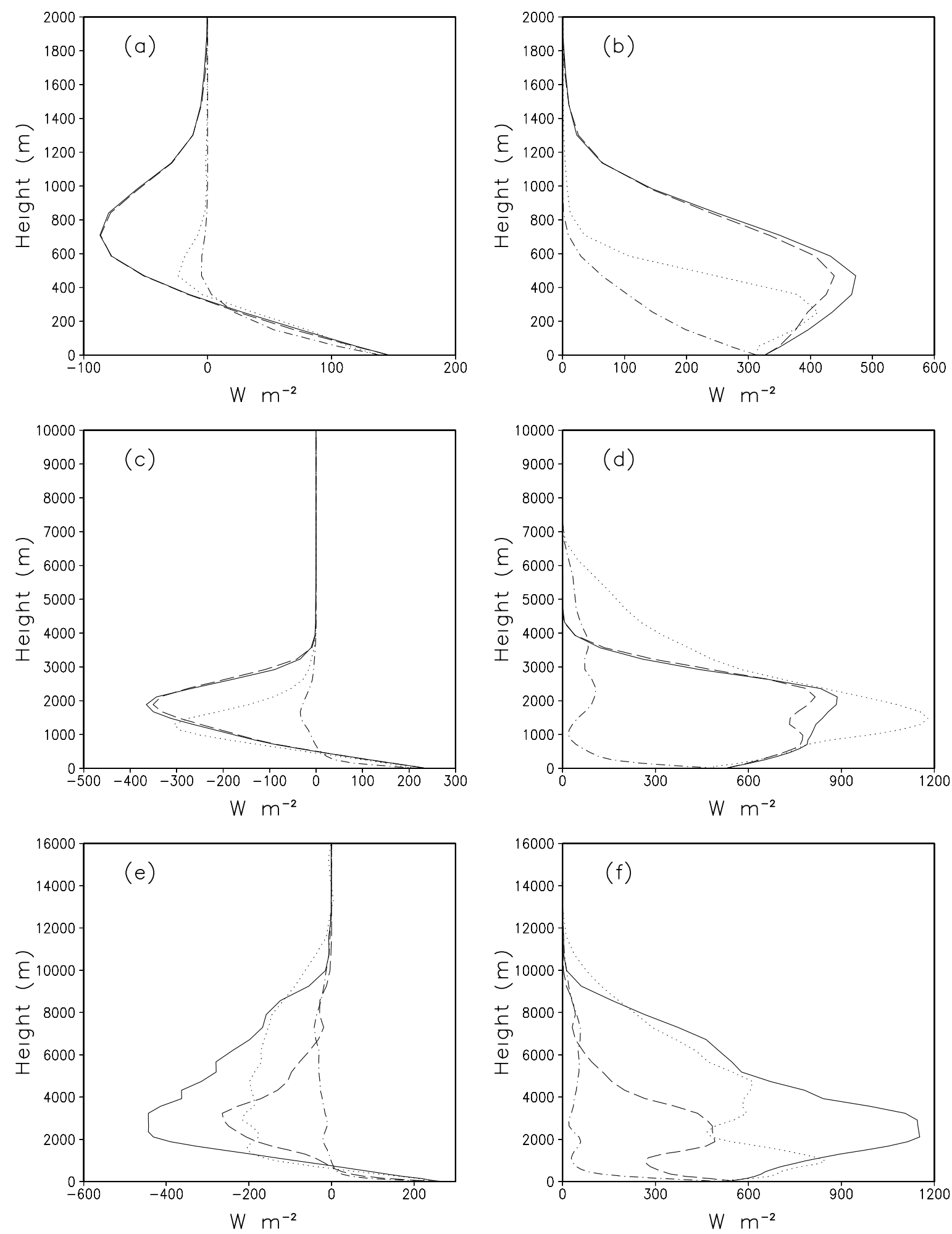